\title{
Taste uncertainty explains developmental effects on susceptibility to peer influence in adolescence
}

Andrea M.F. Reiter ${ }^{1,2}$, Michael Moutoussis ${ }^{1,2}$, Lucy Vanes ${ }^{1,2}$, Rogier Kievit ${ }^{1,3}$, Edward T. Bullmore ${ }^{4}$, lan M. Goodyer ${ }^{4}$, Peter Fonagy ${ }^{5}$, Peter B. Jones, NSPN Consortium ${ }^{6}$ \& Raymond J. Dolan ${ }^{1,2}$

${ }^{1}$ Max Planck UCL Centre for Computational Psychiatry and Ageing Research, University College London, London, UK.

${ }^{2}$ Wellcome Centre for Human Neuroimaging, University College London, London, UK

${ }^{3} \mathrm{MRC}$ Cognition and Brain Sciences Unit, Cambridge, UK

${ }^{4}$ Department of Psychiatry, University of Cambridge Clinical School, UK

${ }^{5}$ Department of Clinical, Educational and Health Psychology, University College London, London, UK

${ }^{6}$ Please see S-table 4 for Consortium membership

\section{Corresponding Author}

Andrea Reiter

Wellcome Centre for Human Neuroimaging, University College London

12 Queen Square

London WC1B5EH, UK

\section{Email}

AMF: a.reiter@ucl.ac.uk

MM:m.moutoussis@ucl.ac.uk

LV: lucy.vanes@ucl.ac.uk

RK: rogier.kievit@mrc-cbu.cam.ac.uk

ETB: etb23@medschl.cam.ac.uk

IMG: ig104@cam.ac.uk

PF:p.fonagy@ucl.ac.uk

PBJ: pbj21@cam.ac.uk

RJD: r.dolan@ucl.ac.uk 
Author contributions: All authors designed research, the NSPN consortium performed research, AMFR and MM analysed the data, RK and LV contributed analytic tools, AMFR wrote the paper, all authors provided significant revisions.

Acknowledgements: The Wellcome Trust funded the 'Neuroscience in Psychiatry Project' (NSPN). All NSPN members (S-Table 4) are supported by a Wellcome Strategic Award (ref 095844/7/11/Z). Ray Dolan is supported by a Wellcome Investigator Award (ref 098362/Z/12/Z). The Max Planck - UCL Centre for Computational Psychiatry and Ageing is a joint initiative of the Max Planck Society and UCL. M. Moutoussis receives support from the NIHR UCLH Biomedical Research Centre. R.A.K. is supported by a Sir Henry Wellcome Trust Grant 107392/Z/15/Z and MRC Programme Grant SUAG/014 RG91365. E Bullmore is in receipt of an NIHR Senior Investigator Award. P. Fonagy is in receipt of a National Institute for Health Research (NIHR) Senior Investigator Award (NF-SI-0514-10157). P. Fonagy was in part supported by the NIHR Collaboration for Leadership in Applied Health Research and Care (CLAHRC) North Thames at Barts Health NHS Trust. The views expressed are those of the authors and not necessarily those of the NHS, the NIHR or the Department of Health. We are grateful to the NSPN management and research assistant teams. 


\section{Abstract}

2 Adolescence is a time of pronounced cognitive, neural, and social change.

3 Adolescents are prone to social influence from peers, with implications for 4 development, both adaptive and maladaptive. However, the underlying cognitive mechanisms of this influence, as well as their neuro-developmental correlates and 6 real-life social consequences are poorly understood. Here, we replicate a crosssectional effect of more susceptibility to peer influence in adolescents in a large dataset of 14 to 24 years old. Crucially, we extend this finding by adopting a

Adolescence, peer influence, social development, taste uncertainty, myelination longitudinal perspective, showing that a within-person susceptibility to social influence decreases over a 1.5 years follow-up time period. Exploiting this longitudinal design, we show that susceptibility to social influences at baseline predicts an improvement in peer relations over the follow-up period. Using a Bayesian computational model, we provide novel mechanistic insight into these effects, showing that in younger adolescents a greater tendency to adopt others' preferences arises out of a higher uncertainty about their own preferences (a phenomenon called 'taste uncertainty'). This taste uncertainty decreases over time and, in turn, leads to a reduced susceptibility of one's own behaviour to an influence from others'. Neurodevelopmentally, we show that a measure of myelination within medial prefrontal cortex, estimated at baseline, predicts a developmental decrease in taste uncertainty at follow up. Thus, using computational and neural evidence, we reveal adaptive mechanisms underpinning susceptibility to social influence during adolescence. 


\section{Significance}

30 Adolescents are particularly susceptible to peer influences, and this can lead to maladaptive behaviours such as binge-drinking or unprotected sexual intercourse (1, 2 ), often linked to impulsivity. Assessing impulsivity with a measure of temporal discounting in a large longitudinal study, we show that younger teenagers are more susceptible to social influence. However, this susceptibility to social influence is not associated with maladaptive real-life behaviours, but instead predicts an increase in quality of real-life social relations. Using computational modelling, we identify a mechanism underlying this higher susceptibility to social influence: younger adolescents' higher uncertainty about what they like drives them to adopt peers' behaviour more strongly. As they grow older, such 'taste uncertainty' decreases, diminishing this susceptibility to social influence. At a neural level, a measure of myelination within the medial prefrontal cortex predicted the observed developmental decrease in taste uncertainty. 


\section{Introduction}

Across many species adolescence is a key period for social development (3). Animal and human studies suggest social interactions are more salient for adolescents than for adults (4). Adolescence is also a period of enhanced susceptibility to peer influence (5-8), an effect which remains highly relevant in the digital age, where adolescents are increasingly exposed to a range of social media (9). Higher susceptibility to social influence is traditionally thought to have particular relevance for the emergence of psychopathology and health damaging real-life behaviours (2, 10-14). Thus, adolescents smoke and drink more alcohol when in the presence of peers, and peers' substance consumption is a predictor of a teenager's own substance use $(13,15,16)$. The prevalence of suicidal and self-injury behaviours, as well as unprotected sexual intercourse, are often related to a social contagion effect during adolescence $(1,17$, 18). However, the directionality of such associations is not clear and alternative accounts frame susceptibility to social influence during adolescence in a less maladaptive context.

It is important to recognise that social influence can change behaviour for the better, an effect widely used for adaptive ends both in education and psychotherapy. Susceptibility to peer influence can be associated with higher psychosocial functioning in young adolescents (8), while an enhanced impact of social influence is observed in neuro-typically developing, but not autistic, teenagers (19). These findings suggest that a higher tendency to integrate social influence into one's own decisions might be an adaptive ingredient in healthy social development during adolescence, a period of life characterised by a shift in social orientation away from the parents towards one's peer group.

Although peer influences on decision-making during adolescence have been widely investigated, several important questions remain unanswered. Firstly, claims on social susceptibility and its real life consequences in adolescents mostly rely on crosssectional designs and modest sample sizes. Here, we applied a longitudinal design in a large cohort of adolescents and young adults to study peer influence on a well characterised task measuring delay discounting - a key measure of temporal impulsivity relevant for development and psychopathology $(20,21)$. Longitudinal designs are important for addressing developmental questions as they provide a basis 
for disentangling cohort or sampling effects from developmental trajectories. We show that social susceptibility not only decreases with age cross-sectionally, but also longitudinally. Longitudinally, we demonstrate that susceptibility to peer influence at baseline predicts the quality of peer relationships over follow-up, highlighting an important adaptive role of social susceptibility in healthy adolescents.

Secondly, though previous studies have established higher conformity towards peers in adolescents than in adults, it remains unclear why this is the case. The adult conformity literature suggests two distinct routes towards conformity, namely informational influences ('copy-when-uncertain', observing others to gain information regarding behaviours that are currently adaptive) and normative influences (adhering to social norms / expectations of the other, bringing direct benefits through belonging, social tension reduction and acceptance $(22,23)$. Many findings on peer influence in adolescent psychology are implicitly interpreted within the framework of normative influence, suggesting the pursuit of social acceptance and sensitivity towards social evaluation by peers is a significant determinant of adolescent decision-making $(3,5$, 10, 24). Here, we tested an alternative hypothesis, namely that informational influences underlie higher conformity in adolescence. To this end, we built on our previously validated (Bayesian) probabilistic reasoning model (25) that describes conformity as a learning effect. In brief, If people are uncertain about exactly what to like ('taste uncertainty'(26)), they can learn about what tastes to adopt by adopting the tastes of their peers'. Using this model, we show that such taste uncertainty decreases both with age and over the course of our longitudinal follow-up, and longitudinal change is strongest in the youngest of our sample. Crucially, both cross-sectional and longitudinal developmental effects on social susceptibility are explained by developmental changes in taste uncertainty, suggesting that higher taste uncertainty in younger adolescents is a key mechanism facilitating peer influence in teenagers.

Thirdly, we were interested in the co-development of brain structures that are relevant for the expression of taste uncertainty. We previously found the medial prefrontal cortex (mPFC) mediating the influence of others' preferences (27). Building on this, and using novel in-vivo myelin-sensitive magnetization transfer MRI (28), we identify a myelin marker in medial prefrontal cortex that predicts a developmental decrease in taste uncertainty over our longitudinal follow-up period. 


\section{Results}

109 To probe a susceptibility to social influence, we used a social version of a delay 110 discounting task (Figure 1). In short, this task allows us to measures a person's 111 temporal discounting coefficient (how much less a future reward is worth, depending 112 on the delay of its delivery) as well as changes in their discount function pre vs. post 113 learning about someone else's discount preferences (See Figure 1, Supplementary 114 Methods and $(25,27,29)$ for details). Here, we defined susceptibility to social influence 115 as the degree of change in one's own discount rate towards the preference of a social 116 partner, following exposure to the preferences of a social partner. 
a

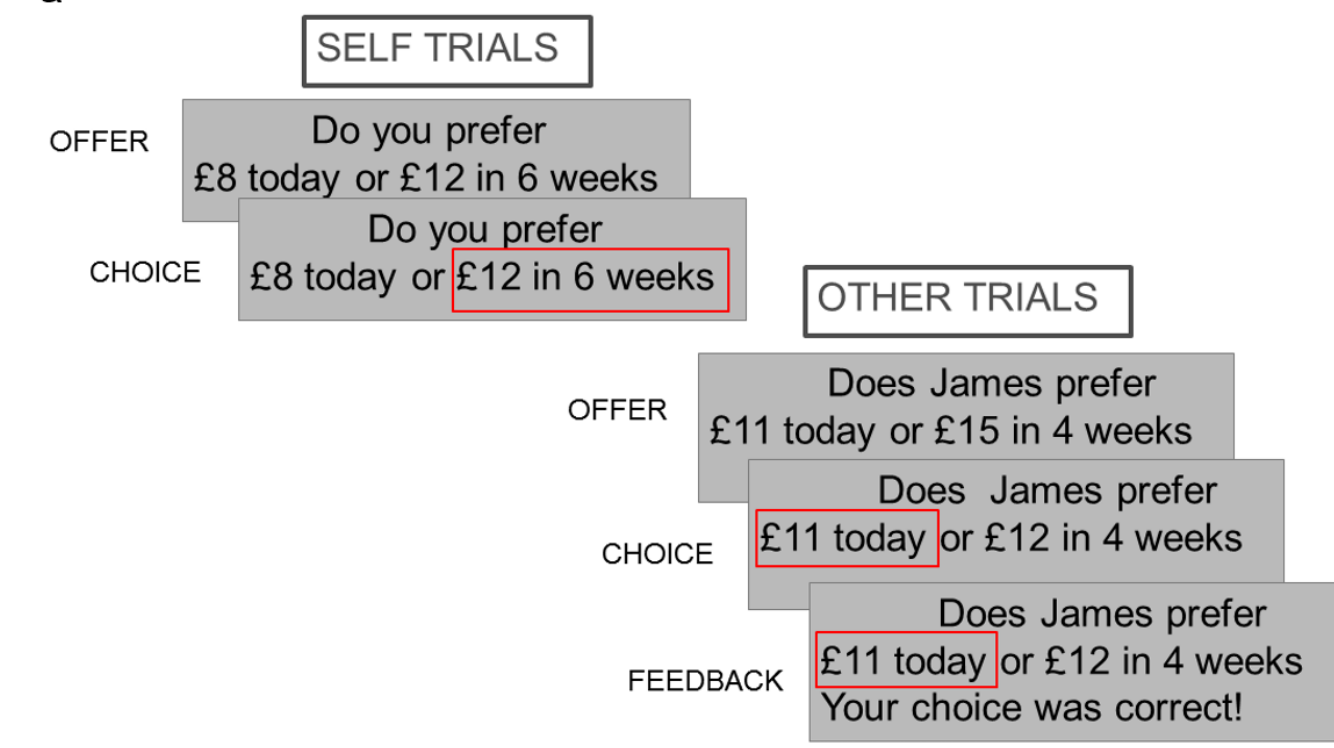

b

Phase1

SELF

60 trials
Phase 2

OTHER

max. 60 trials
Phase 3
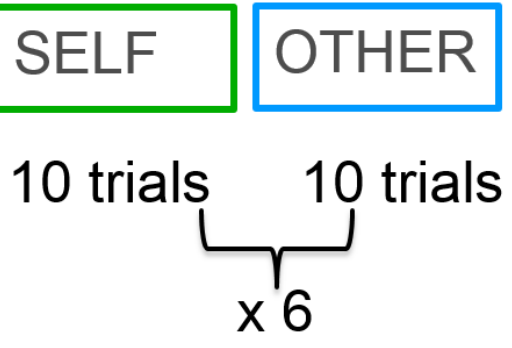

Figure 1. Social Delay Discounting Task. A) Example trial for "self" and "other" trial types. In self trials, participants see an offer of a smaller amount of money they can receive on the same day or a larger amount of money they can receive after a variable delay period. Subjects were instructed to indicate their preference according to their true personal taste and, to enforce expression of true preferences, they were told that one trial would be chosen at random to determine their pay out. In "other" trials, subjects chose between the smaller, immediate and the larger, delayed option on behalf of another person, and received feedback on these choice thereby enabling them to learn the others' delay discounting preferences. 


\section{Development of susceptibility to social influence}

Cross-sectional baseline age effects

At baseline, we observed a significant negative association between social susceptibility (indexed as the change in discount rate upon learning about the discount rate of another agent) and age, such that social influence declined with age ( $r=-.10$, $\mathrm{df}=782, \mathrm{t}=-2.94, \mathrm{p}=.003$, Figure $2 \mathrm{a}$ ). Age explained about $1 \%$ of the variance in social susceptibility. Though this is significant, it is substantially lower than what has been observed in previous reports, as is often the case with larger, rigorously controlled, replication studies (30).

Longitudinal analysis

We tested whether social susceptibility also changed intra-individually, within the 1.5 years follow-up period. Indeed, a factor time (baseline vs. follow-up) significantly predicted social susceptibility in a linear mixed effects model $(F(1,566.64)=5.11$, $p=.02$ ), along with baseline age. Social susceptibility decreased intra-individually over the 1.5 years follow-up period. The interaction of baseline age with time was not nominally significant $(F(1,568.31)=3.78, p=.05)$. Although not nominally significant, suggests that intra-individual change in susceptibility was most pronounced in the youngest subgroup of the sample.

Retest Subsample - Testing for training vs. developmental effects in a 6-month follow up subsample of participants

To determine whether the observed longitudinal differences were predominantly due to retest effects or development, we examined a sample of participants who were also tested 6 months apart ('short follow-up', judged to be a short time with respect to maturation). This comprised a sub-sample of $n=55$ of the total group who came to the lab three times (baseline, 6-month 'short' follow-up, 1.5-years 'long' follow-up), in the same manner as per our main sample (see Methods). Repeating the same analysis of longitudinal effects on social susceptibility in this short follow-up sample, we observed a significant effect of time point (baseline, short follow-up, long follow-up) on social susceptibility $(F(2,104.926)=6.87, p=.002)$. Post-hoc analysis showed that 
159 susceptibility decreased significantly over the 18-month period $(t=3.47, p<.001)$, and 160 between 6 and 18 month of follow-up ( $t=2.88, p=.005)$, but critically did not do so over 161 the 6-month period from baseline $(\mathrm{t}=.59, \mathrm{p}=.56)$. This pattern does not support a mere 162 training effect, as if this was the case we would expect a stronger change after 6 163 months than after 18 months. 
a

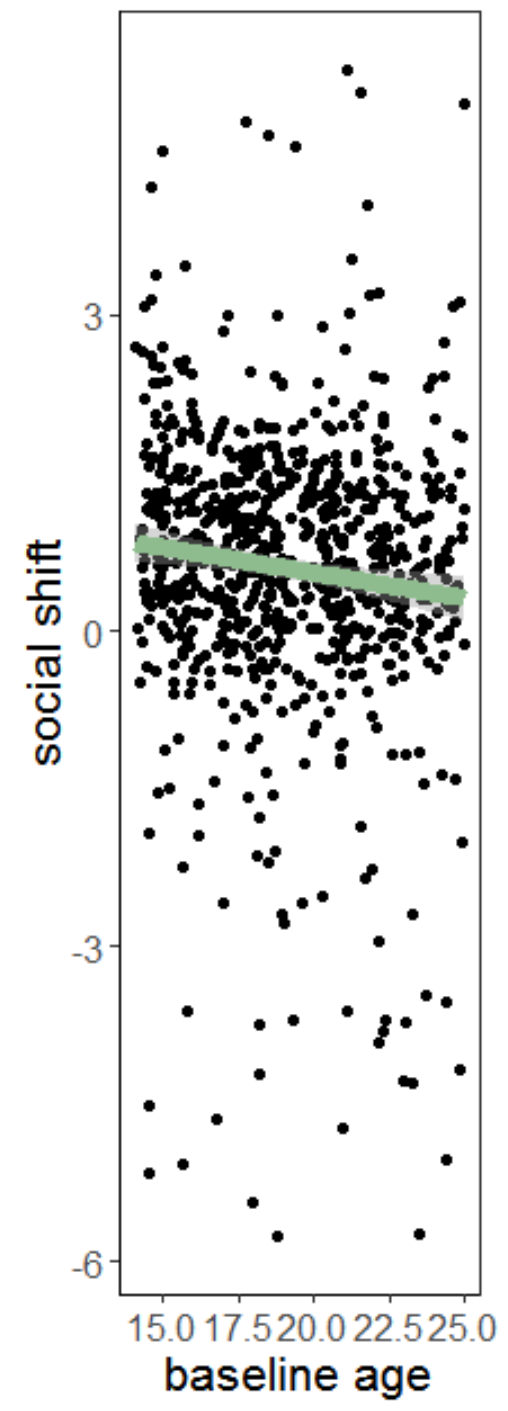

b

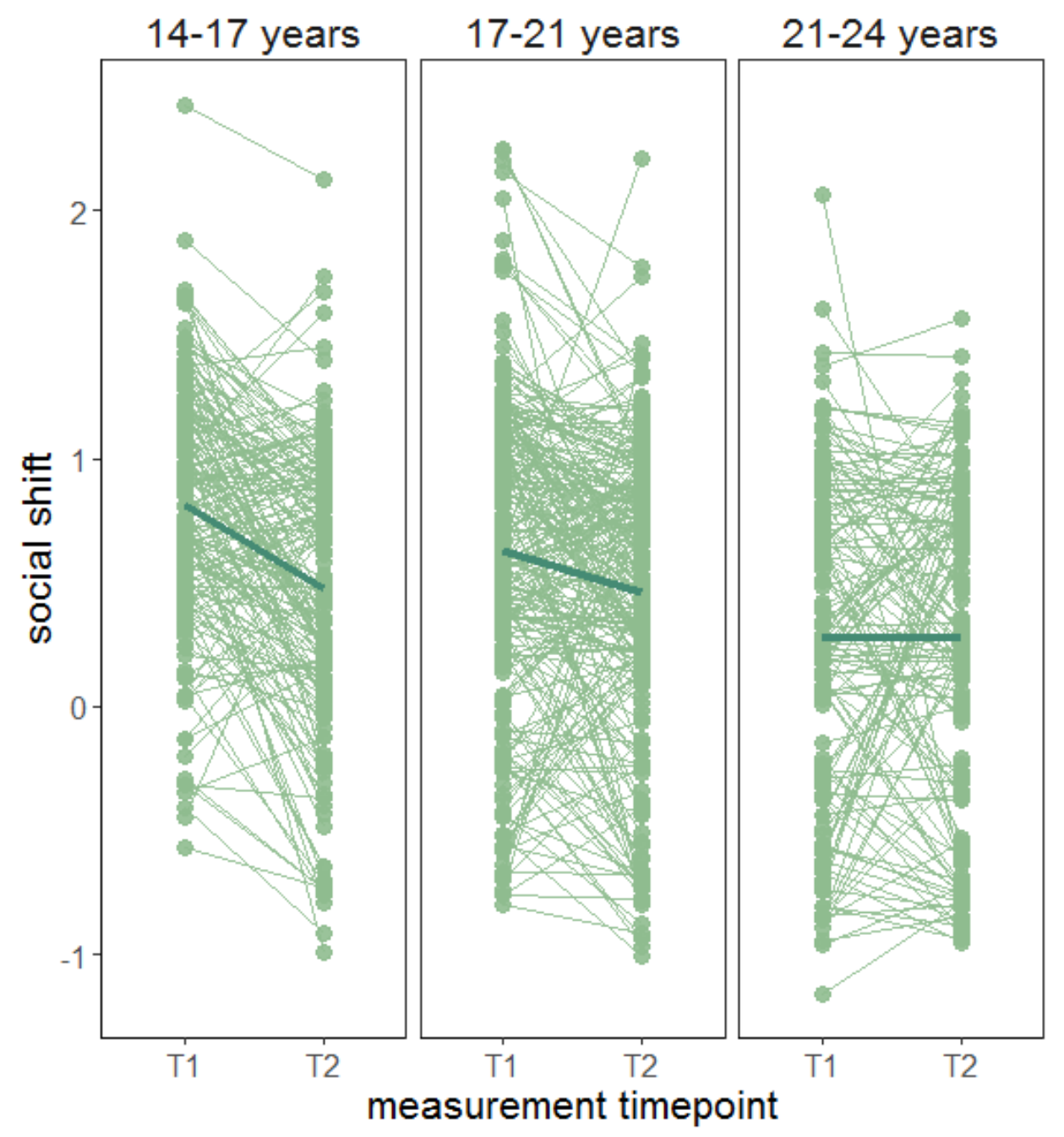

Figure 2. Developmental effects on social susceptibility. A) A tendency to show a peer-induced shift in delay discounting preferences (positive values indicate a change towards the partner) declines with age. B) Susceptibility to peer influence also decreases within person over the course of the longitudinal follow-up period. The longitudinal change depends on baseline age and is more pronounced in younger participants. We plot posterior estimates from our mixed effects model. Note that age entered the model as a continuous regressor, here we plot 4-year-age bins $\leq 17$ years 


\section{Relationship with the development of psychosocial functioning}

In previous work, we observed a positive association of susceptibility to peer influence and sociability in a smaller cross-sectional study, including young adolescents(8). Here we tested for this association again, including in a longitudinal manner, in our larger dataset. We used a bivariate latent change score model (31) to test for a codevelopment of social susceptibility and quality of peer relationships, as measured by the Cambridge Friendship Questionnaire (CFQ(32, 33)) The model showed that the perceived quality of peer relations increased from baseline to long follow-up (significant intercept of the latent CFQ change score: $z=2.28, p=.022$, standardised estimate $=.09$ ). There was no significant covariation of social susceptibility and peer relations at baseline $(\mathrm{z}=-.77, \mathrm{p}=.44$, standardised beta $=.03)$. However, the latentchange model revealed a small, but significant, positive association between social susceptibility at baseline on rate of change in peer relation development from baseline to long follow-up. In effect, those who showed a higher tendency to shift their preferences towards their partners' also reported larger gains in social integration from baseline to long follow-up $(z=2.12, p=.03$, standardised beta $=.08)$.

Previous findings $(6,8)$ show that developmental effects on social susceptibility might be particularly pronounced in younger teenagers. Thus, we repeated the path analysis separately on age specific subsamples of our main sample, namely a younger (participants who were $<18$ at both baseline and long follow-up, $n=116$ ) and an older (participants who were adults, i.e. $\geq 18$ years old, at both measurement time points, $\mathrm{n}=248$ ) subsample. Comparing this model to a model where the path of interest (social susceptibility at baseline $\rightarrow$ quality change in peer relations at long follow-up) was constrained to be equal between the younger and older subsample, revealed a significant advantage of fitting an age-dependent sub-group-specific parameters (Log likelihood Ratio Test, $\Delta \mathrm{x}^{2}=6.20, \Delta \mathrm{df}=1, \mathrm{p}=.01$ ). This indicates differences in a younger vs. older subgroup regarding the degree to which social susceptibility at baseline influences real life social development.

Analysing the path of interest separately for the younger ( $<18$ years) and older ( $\geq 18$ years) subgroup revealed a significant effect of social susceptibility on social development in the adolescent ( $<18$ years) group alone $(\mathrm{z}=2.31$, standardised beta $=.23, p=.02$, see Figure 3 ), whereas there was no significant coupling of social susceptibility on social development in the young adult $(<18$ years) group $(z=-.06$, 
213 beta=.003, $p=.96$ ). This suggests that greater susceptibility to social influence earlier

214 in adolescence might be an important factor affecting development of integrative social 215 relationships as we grow up. Subsequently, we repeated the structural equation model 216 to analyse an association with selected maladaptive traits, which had been discussed 217 in the context of delay discounting and peer influence in the literature (2, 34-36), 218 namely externalising behaviours, and alcohol consumption, both assessed using the 219 behaviours checklist questionnaire (37). In neither of these models did we find an 220 association of social susceptibility with maladaptive behaviours (all standardised betas $221 \leq|.11|$, all $z s \leq|1.27|$, all ps $>$.203). 
a

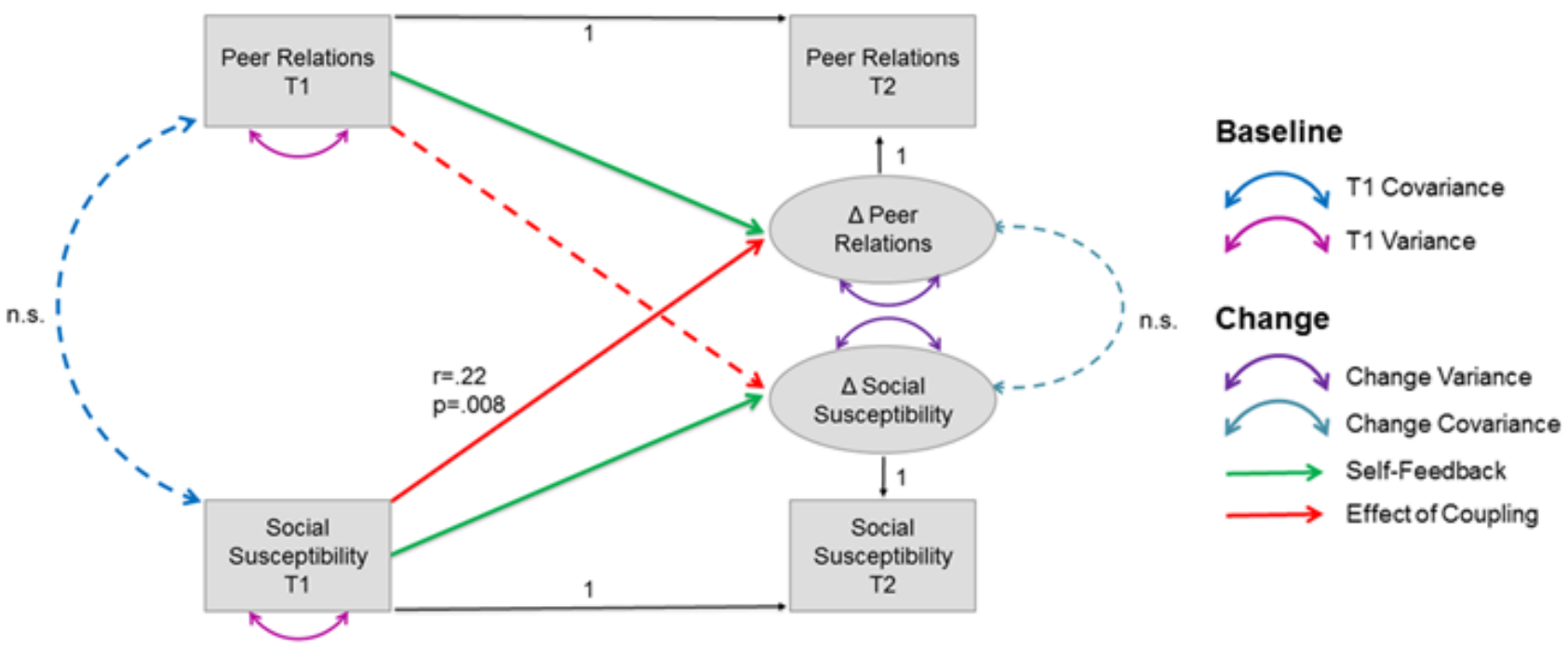

b

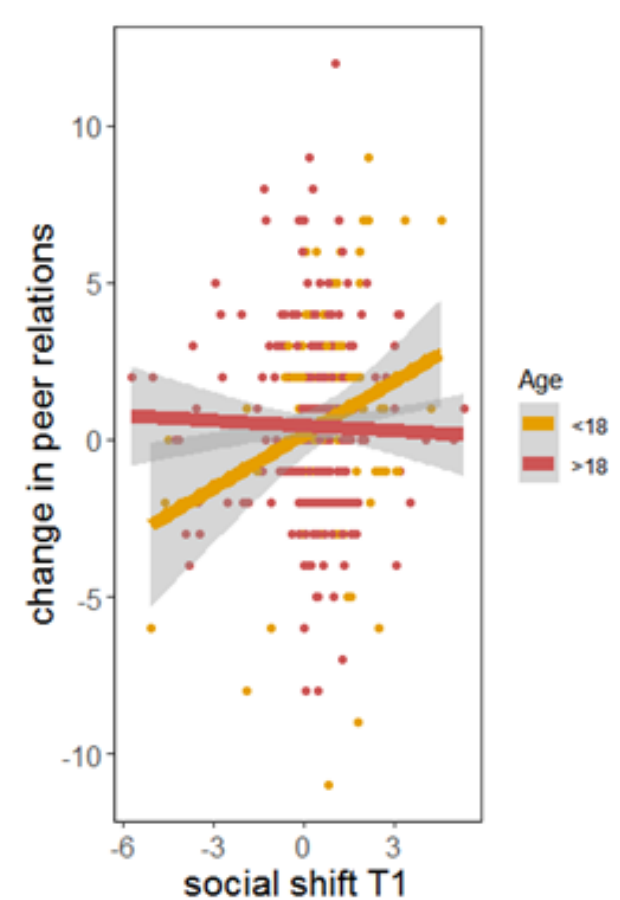

C Development:

Quality of Peer Relations

in Adolescents

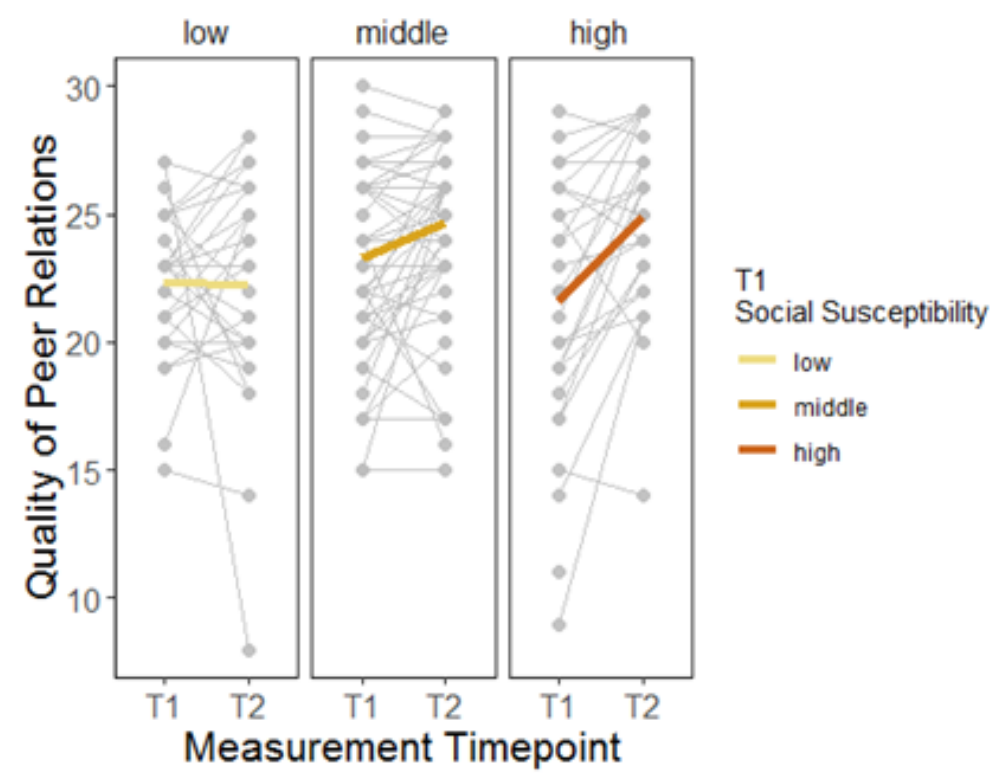

Figure 3. A) Latent change score model of the longitudinal development of social susceptibility in our task, the longitudinal development of real-life psycho-social functioning (perceived quality of peer relations) from baseline to long follow-up ( 1.5 years later) as well as their co-development. Social susceptibility at time point 1 significantly predicts an increase in the quality of peer relationships within the followup period. Solid lines: significant path, dashed line: non-significant path. B) This positive association was driven by the younger ( $\leq 18$ years old) people in our sample, but was not significant in those aged 18 or older. The full set of parameter estimates is included in supplementary table 1. C) Change in the Quality of Peer Relations, plotted as a function of T1 Social susceptibility. 
236 One reason for conformity is informational influence, whereby humans use 237 observational information to reduce uncertainty about what to like, even if, as in our task, this does not produce immediate material benefits $(16,19)$. We tested the hypothesis that the observed developmental reductions on social susceptibility occur as a consequence of developmentally decreasing uncertainty about own preferences ("taste uncertainty"). Thus, in a next step, we used a previously validated formal computational model (19) to estimate an individual 'taste uncertainty' parameter (see supplementary results for effects of other parameters of the model). This allowed us to test for developmental effects on taste uncertainty and whether this accounts for the observed susceptibility to social influence.

\section{Computational modelling: Developmental Effects on taste uncertainty}

248 Taste uncertainty predicts susceptibility to social influences

249 Taste uncertainty was significantly associated with social shift at both measurement 250 time points, consistent with an informational account of conformity (all $r>50$, all $251 \mathrm{t}>15.57$, all $p s<2.2 \mathrm{e}-16$, see S-Figure 1).

252 Cross-sectional baseline age effects

253 At baseline, taste uncertainty negatively correlated with age $(r=-.16, t=-4.56, d f=780$ $\mathrm{p}=5.869 \mathrm{e}-05$, Figure 4a).

\section{Longitudinal Analysis}

256 A linear mixed effects model revealed a significant effect of baseline age $257(\mathrm{~F}(1,564.53)=14.22, \mathrm{p}<.001)$, time $(\mathrm{F}(1,564.86)=5.71, \mathrm{p}=.017),(\mathrm{F}(1,564.53)=5.71$, $258 \mathrm{p}=.017)$ and an interaction of baseline age and time $(F(1,565.41)=9.29, p=.002)$ on taste uncertainty. To visualise the latter interaction, we plot longitudinal (intraindividual) change as a function of age at baseline in Figure 4b. Figure 4b suggests that relevant longitudinal changes in taste uncertainty were strongest in those that were $\leq 17$ years of age at baseline. 
a

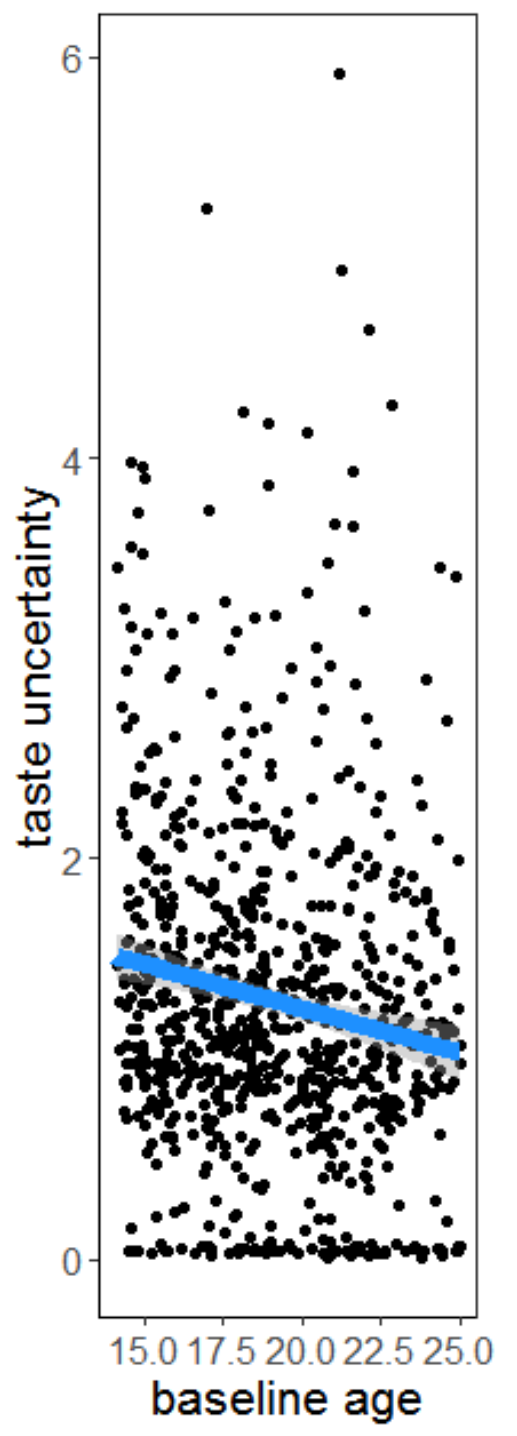

b

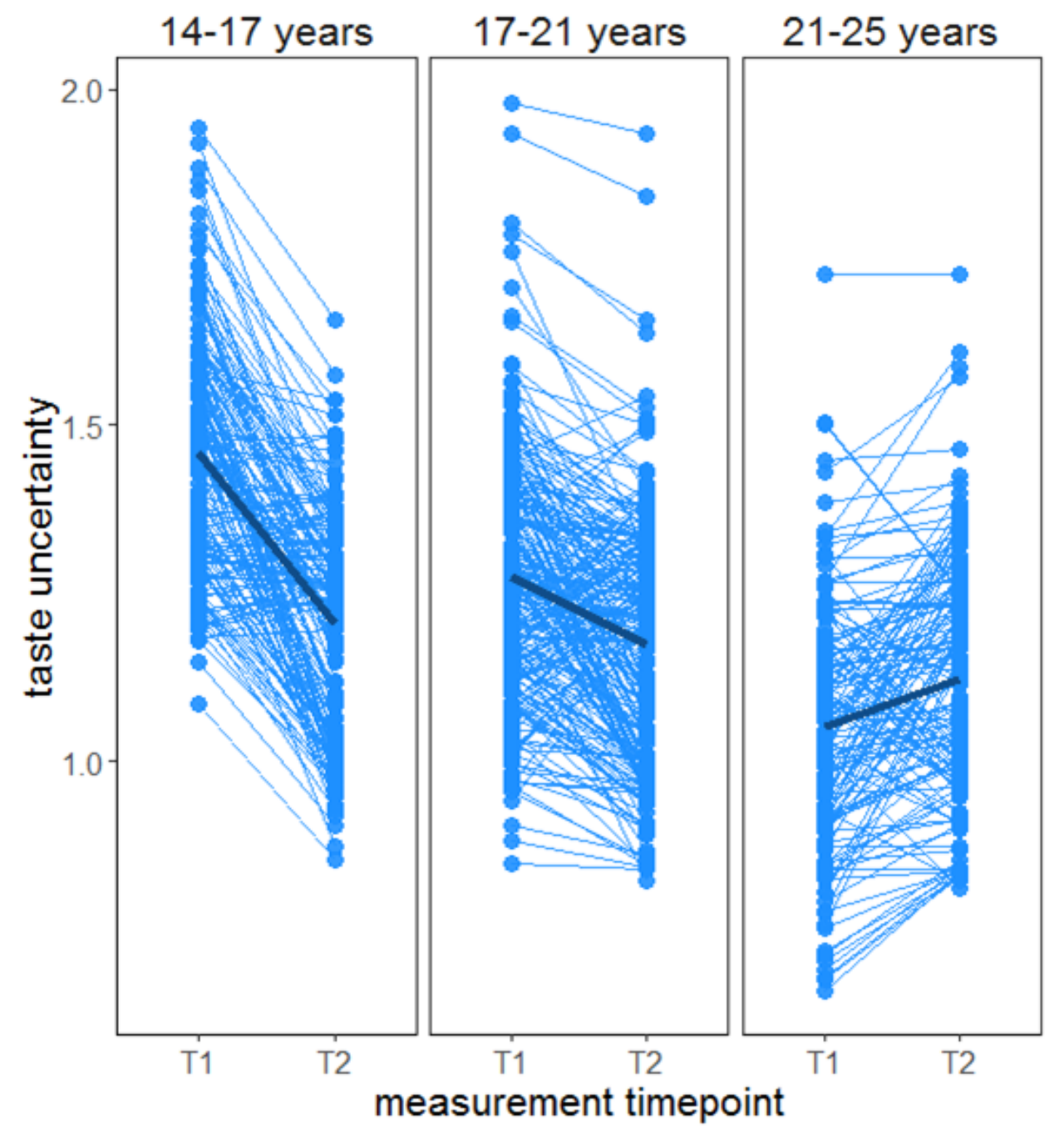

Figure 4. Taste uncertainty decreases cross-sectionally (panel a) and over the 1.5 years follow-up (panel b). This decrease is most pronounced in the youngest participants. We plot posterior estimates of our mixed model analysis. Note that age entered the model as a continuous regressor, here we plot 4-year-age bins $\leq 17$ years 271 old, $>17 \leq 21$ years old, $>21$ years old), only for visualization purposes. 
272 Retest Subsample - Testing for training effects in a 6-month follow up subsample of participants.

274

In line with findings in our main study sample, we observed a significant effect of time on taste uncertainty $(F(1,105.29)=3.57, p=.032)$ in the 'short follow-up' subsample. Post-hoc inspection revealed that taste uncertainty decreased within-person over the three measurement time points in the reduced subsample. Post hoc tests showed that a contrast of first and last (1.5 years follow-up) time points was significant ( $\mathrm{t}=2.670$, $\mathrm{p}=.009)$, whereas the change between first and 6 -month follow-up was not $(\mathrm{t}=1.23$, $\mathrm{p}=.220$ ). This pattern of findings is inconsistent with changes being due to a mere training effect, as we would expect stronger change after 6 compared to 18 months.

\section{Mediation analyses: Taste uncertainty explains developmental effects of social susceptibility}

Our computational model of social shifts posits a specific mechanism by which social influence arises, namely a reduction in one's own taste uncertainty by learning how someone else performs a task $(22,25)$. As taste uncertainty declines with age, and decreases longitudinally within person, this raises a possibility that age-related changes in social susceptibility (as found here and in previous studies) is driven by the age-related change in taste uncertainty. To test this, we first set up a model where we tested a possible mediation of the cross-sectional age effects on preference shift by taste uncertainty on baseline (baseline) data. We found that an effect of age on preference shift was accounted for by the mediating effect of taste uncertainty, corresponding to a significant full mediation (significant proportion of mediation (38): estimate $=.72, z=3.03, p=.002$, Figure $5 \mathrm{~A}$, Table 1 ). That is, the significant age effects on social susceptibility, as found here and in many previous studies, are, in this study, explained by age effects on taste uncertainty.

In a next step, we examined the covariation of longitudinal change in taste uncertainty with longitudinal change in social susceptibility, using latent change score modelling. To do so, we changed the autoregressive and coupling effects to co-variances, to display and model the unconditional change scores. We observed a significant covariation of rates of change in both parameters (raw beta=.41, standardised beta $=.21, z=3.82, p<.001$ ), in line with our assumption that development of social susceptibility is accounted for by development of taste uncertainty. 


\begin{tabular}{|c|c|c|c|c|c|c|c|c|}
\hline path & notation & $\begin{array}{c}\text { est } \\
\text { (unst) }\end{array}$ & se & $\mathbf{z}$ & $\mathbf{p}$ & $\begin{array}{c}\text { lower } \\
\text { Cl }\end{array}$ & $\begin{array}{c}\text { upper } \\
\text { Cl }\end{array}$ & $\begin{array}{c}\text { est } \\
\text { (stand) }\end{array}$ \\
\hline $\begin{array}{c}\text { social } \\
\text { susceptibility } \\
\text { age }\end{array}$ & c' & -0.01 & 0.02 & -0.86 & 0.39 & -0.04 & 0.02 & -0.03 \\
\hline $\begin{array}{c}\text { social } \\
\text { susceptibility } \\
\text { taste uncertainty }\end{array}$ & b & 0.80 & 0.07 & 11.82 & $<.001$ & 0.66 & 0.93 & 0.50 \\
\hline $\begin{array}{c}\text { taste uncertainty } \\
\text { age }\end{array}$ & a & -0.05 & 0.01 & -4.37 & $<.001$ & -0.06 & -0.02 & -0.15 \\
\hline $\begin{array}{c}\text { indirect } \\
\text { (mediation) path }\end{array}$ & a*b & -0.04 & 0.01 & -4.08 & $<.001$ & -0.05 & -0.02 & -0.08 \\
\hline total & $c^{\prime}+\left(a^{*} b\right)$ & -0.05 & 0.02 & -2.71 & .007 & -0.08 & -0.01 & -0.11 \\
\hline $\begin{array}{c}\text { proportion } \\
\text { mediated }\end{array}$ & $\begin{array}{c}\text { indirect/tot } \\
\text { al }\end{array}$ & .72 & .24 & 3.03 & .002 & .255 & 1.19 & 0.72 \\
\hline
\end{tabular}

Table 1. Mediation results: T1 data 
a

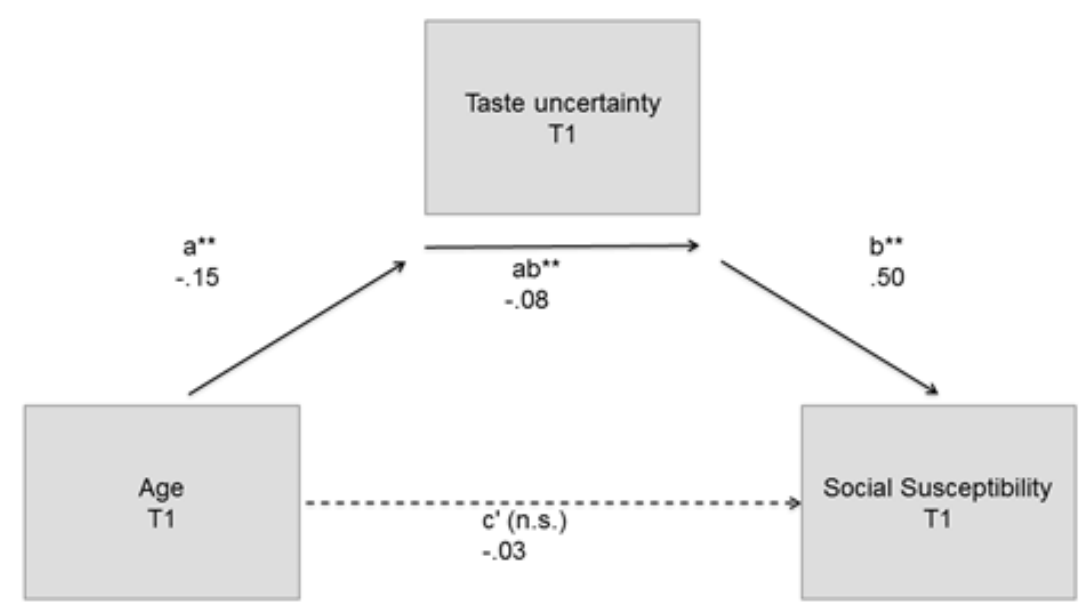

b

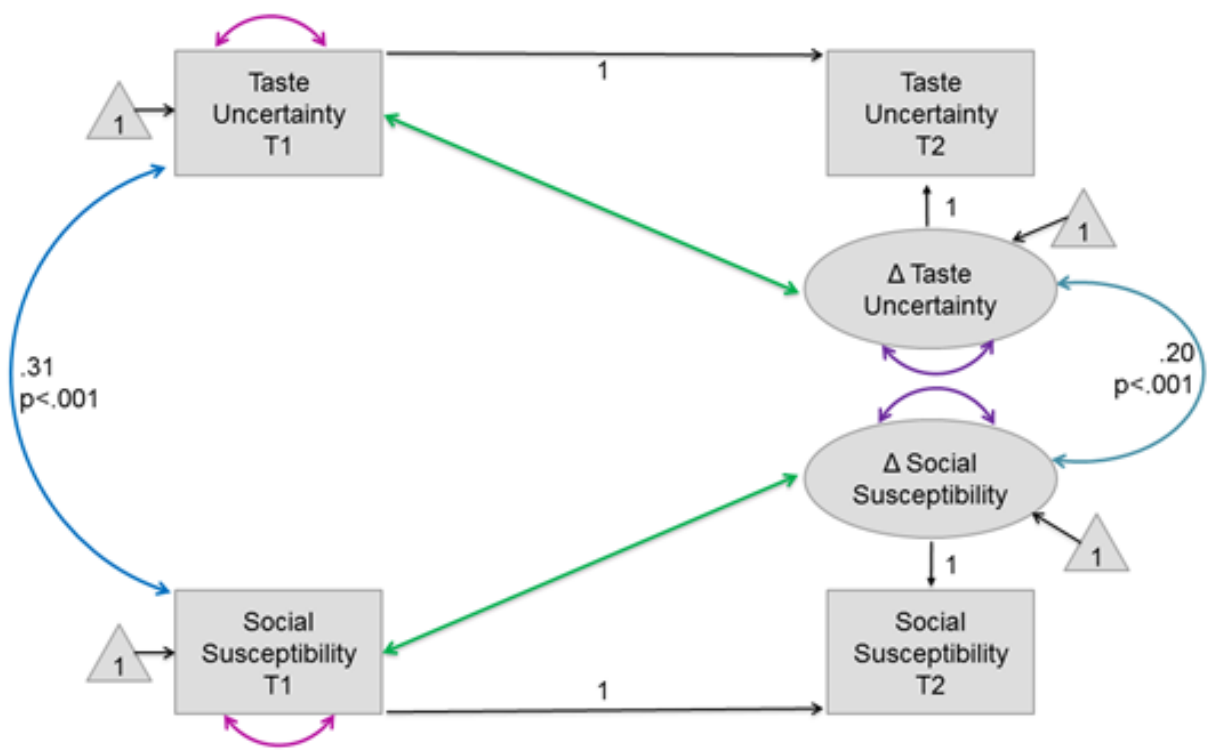

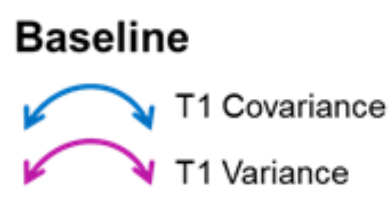

Change

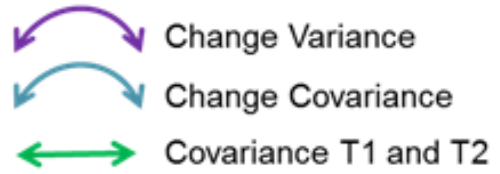

305 Figure 5. A) Mediation analysis for preference shift as predicted from age and mediated by taste uncertainty at T1. Age predicted taste uncertainty (path a). The mediator (taste uncertainty) predicted preference shift (path $b$, controlled for the age effect on taste uncertainty). Importantly, the mediation effect was significant (path ab). The direct path c', namely the age effect on preference shift after accounting for the mediation, was not significant. The proportion of total variance explained by the mediation effect was significant. Thus, the age effect on social susceptibility at baseline was accounted for by taste uncertainty. See table 1 for the full set of parameter estimates including standardised an unstandardised betas. B) In line with our assumption that development of social susceptibility is accounted for by development of taste uncertainty, the bivariate latent change score model not only showed a significant covariation of taste uncertainty with social susceptibility at T1, 
317 but also significant covariation of the rate of change in both domains. The full set of 318 parameter estimates is included in supplementary table 3. 


\section{Co-Development of brain structural correlates and taste uncertainty}

\section{1}

322

323

324

To establish whether there is a structural neural correlate for developmental effects on taste uncertainty, we used bivariate latent change score modelling testing for the codevelopment of cognitive and brain structural development in a subsample of subjects who underwent both our experimental sessions (baseline and 18 months) and structural MRI ( $n=186$, see Methods). Here, we focussed on myelination, given its pivotal role in adolescent brain development, and building on recent findings on its association with trait measures in adolescent development (28). In a similar discounting task, utilizing repetition-suppression to assess neural plasticity, we previously showed that medial prefrontal cortex (mPFC) is the principal region expressing within-task plasticity as preferences shift (27). See supplementary methods for pre-processing of the MRI data.

Using an anatomically defined mPFC mask (based on the Harvard-Oxford atlas, see $\mathrm{SI}$ ), we extracted estimates of a myelin-sensitive marker, Magnetisation Transfer saturation (MT). This allowed us to investigate a cross-domain coupling that captures the extent to which change in one domain is a function of the starting level in the other using latent change score modelling (model fit indices: pchiz=.22, CFI=.96, RMSEA $=.04$, SRMR $=.03$ ). Sex, age, scanning site and general IQ were included in the analysis as covariates (28). We observed that baseline intra-cortical mPFC MT was predictive of the longitudinal change in taste uncertainty (raw beta=-3.92, standardised beta $=.-13, z=-2.12, p=.03$, Figure 6 ), i.e. more intra-cortical mPFC myelin lead to a more pronounced reduction in taste uncertainty over the 1.5 year follow-up. Taste uncertainty did not significantly predict longitudinal brain development, and there was no covariance in rates of change in both domains (all betas $.10 \leq$ all ps $\geq .13$, see S-Table 3 for full output of the latent change score model). Repeating the same structural equation model using estimates of myelin in the visual cortex as a control region (model fit indices: $\mathrm{Chi} 2=.38, \mathrm{CFI}=.99$, $\mathrm{RMSEA}=.02$, SRMR $=.03$ ) (39), we did not observe any cross-domain coupling of our task measure with myelination across development (all beta<.033, all $p>.51$ ). 


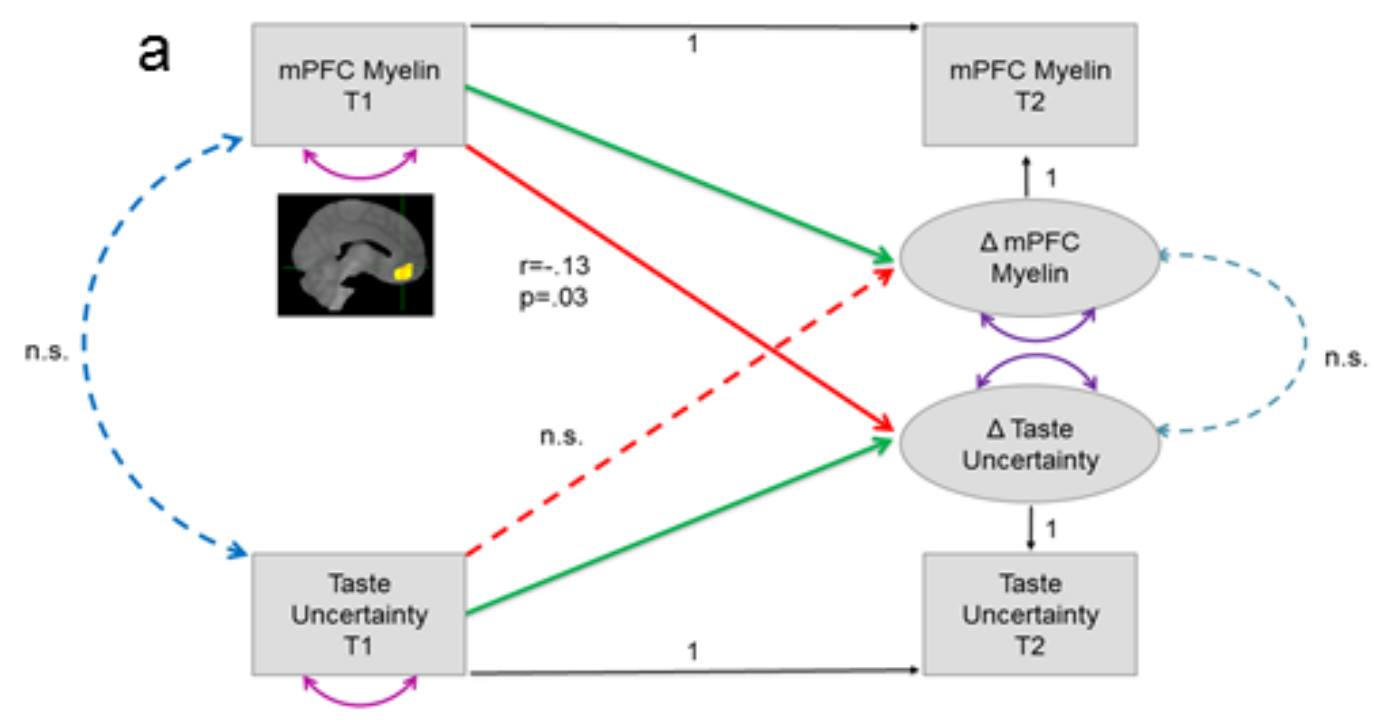

\section{Baseline}

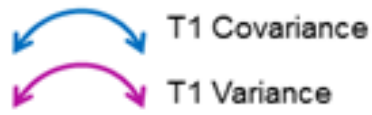

\section{Change}

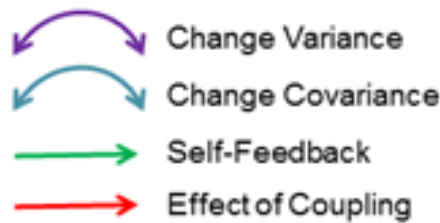

b

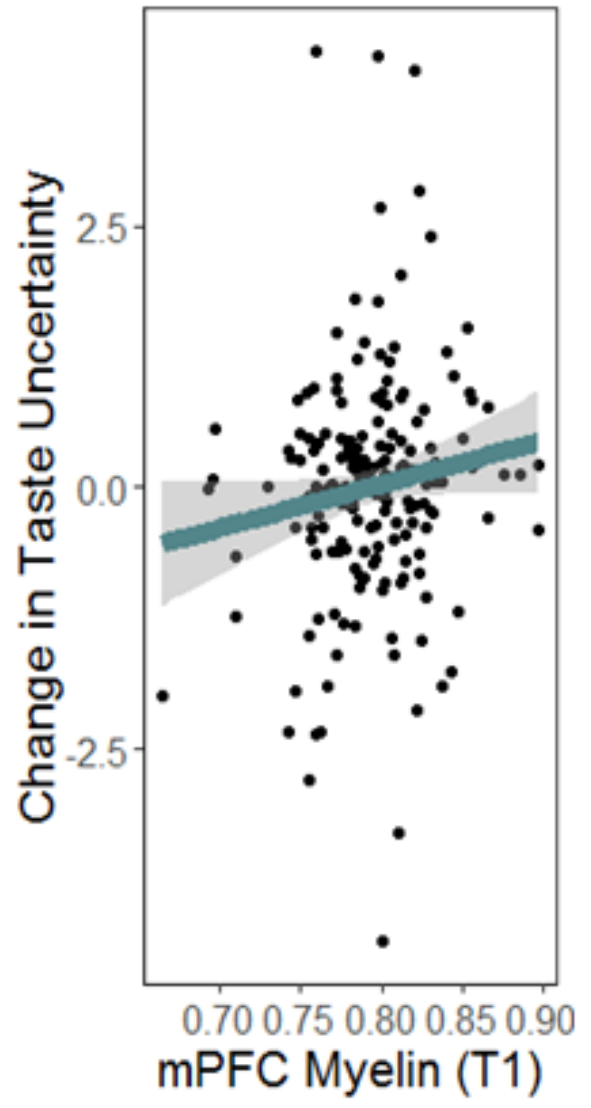

C

T1

Myelin Marker

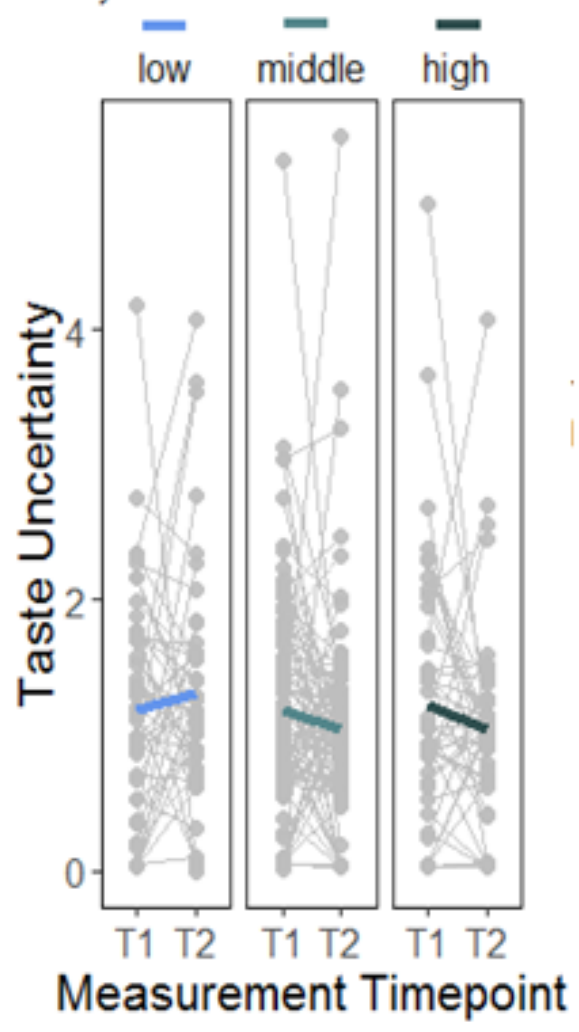

350 Figure 6. A) Bivariate latent change score model. Three developmental brain351 behaviour relationships are possible and tested for by the model: 1) differences in 352 myelin at baseline affect the rate of taste uncertainty decrease; 2) taste uncertainty at 353 baseline predicts the degree of myelin gain between baseline and long follow-up, 3) 354 correlated change (the degree of reduction in taste uncertainty is correlated with the 355 degree of myelin change). While the path indicating the mPFC myelin marker as a significant predictor of longitudinal change in taste uncertainty was significant (beta=.- 
357 13, $p=.03)$, the other cross-domain coupling paths were not. Solid lines: significant 358 path, dashed line: non-significant path. No means are displayed for clarity; the full set 359 of parameter estimates is included in supplementary table 3. B) Higher values of the 360 mPFC myelin marker at measurement timepoint 1 led to a more pronounced 361 longitudinal change in taste uncertainty (note that a stronger longitudinal decline is 362 coded as positive ("more change") for illustration purposes). C) Longitudinal change in Taste Uncertainty as a function of different levels of T1 myelin marker values. 


\section{Discussion}

In adolescence, a greater susceptibility to social influence is considered a driver of maladaptive real-life behaviour (e.g. drinking, reckless driving, delinquency, suicidal behaviours) $(10-13,15,16)$. Here, using a longitudinal design involving a large cohort of 14-24 year-olds, combined with quantitative brain imaging, we replicate a finding of increased social susceptibility in adolescents and characterise its neurodevelopmental and computational basis.

We replicate previous developmental effects on social susceptibility in the paradigmatic case of delay discounting, showing that social susceptibility decreases with age from adolescence to adulthood. We extend on this previous finding in a number of ways. Firstly, we provide longitudinal evidence for a developmental decrease in susceptibility to social influences. Secondly, we show that social susceptibility in young healthy adolescents is adaptive with respect to longitudinal improvements in peer relationships. Thirdly, we outline a cognitive and computational basis for these effects, showing that higher social susceptibility in younger adolescents is best explained by this age defined population being more uncertain about what they like, thereby rendering them more prone to adopt others' preferences. Over the course of development this 'taste uncertainty' decreases, which in turn attenuates a need, and consequential impact of, social influences on one's own behaviour. Lastly, we identify a candidate neuro-developmental correlate of this effect by showing that a myelin sensitive marker within mPFC, a key region mediating social preference shifts, predicts longitudinal change in taste uncertainty.

We replicate our previous finding, from a young adult sample, showing delay discounting preferences can be systematically changed by learning about another's delay discounting preferences $(27,29)$. Extending these findings, we now show that the degree of a preference shift is, in this sample of 14-24 years olds, most pronounced during younger adolescence, a developmental period characterised by significant social-affective transformations (3). Importantly, our longitudinal design allowed us for the first time to demonstrate a within-person developmental decrease in this social susceptibility. A separate analysis in a retest sample showed that these observations could not be explained by a training effect. 
Importantly, we found that such higher susceptibility at a younger age has an important adaptive, rather than maladaptive, role in this healthy population. Exploiting our accelerated longitudinal design $(28,31,33,40)$, we demonstrate that the developmental improvements in real-life psycho-social functioning, assessed by the perceived quality of peer relations after 1.5 years, is predicted by higher susceptibility to social influence, particularly in younger teenagers. This extends cross-sectional findings in healthy younger adolescents which showed that behavioural contagion was associated with a higher degree of social functioning (6). Notably, in previous accounts, susceptibility to social influence was mostly highlighted in the context of maladaptive real-life behaviours in teenagers. For example, both real-life and digital peer influence is suggested to lead to higher rates of delinquency, real-life risk-taking, unprotected sexual intercourse, substance consumption and suicidal behaviours (1, $2,10-16,18)$. In this study of healthy adolescents however, susceptibility to social influences did not relate to externalizing psychiatric symptoms or substance consumption, nor did it predict such potentially maladaptive behavioural tendencies in a longitudinal fashion. This discrepancy might suggest that the impact of higher susceptibility to peer influence in teenagers depends on the very nature of social influence, including the nature of the role models who exert this influence. Being behaviourally responsive to peers may be generally thought of as functional and predictive of resilience in the face of adversity. In our sample of relatively healthy teenagers, it indeed led to successful social adaptation in real life, a key requirement of adolescent development. However, it is equally the case that maladaptive consequences might arise if teenagers have to navigate less advantageous or unstable conditions, where they are likely to be confronted with less desirable role models. For example, as recent models of conduct problems emphasize, sensitivity to peer influence for youth in distressed urban neighbourhoods may carry risk when a teenager overgeneralizes and automatically, without mentalizing, deploys a learnt response (e.g. fighting back aggressively when challenged) in a setting where it is not adaptive, such as in school (41).

Finally, using computational modelling, we provide a mechanistic account for the frequent previous observation of adolescents being more prone to peer influence than adults (e.g. $(5,6,8))$. In principle, two routes to conformity are possible, normative influences (adhering to social norms and expectations to gain interpersonal benefits 
such as being part of a group (16), and informational influences (reducing uncertainties about the world and the self by observing others). In the existing literature, peer influences on decision-making are generally implicitly interpreted within a normative account of conformity. Using a Bayesian model, previously validated on this same task (19), we specifically probed the latter hypothesis. We show that uncertainty about one's own preferences predicted social shift, and that developmental differences in susceptibility to social influences are fully explained by decreasing uncertainty as participants aged. This provides a novel interpretation of our current and previous findings. Intriguingly, it places susceptibility to social influence into an adaptive context - as a rational means to reduce one's own uncertainty.

Future studies of adolescent development may usefully manipulate both informational and normative sources of conformity to disentangle their respective effects on adolescent behaviour, and to test for putative interactions between these processes. For example, is social influence on adolescents highest when they are uncertain and feel an enhanced need to conform in order to be accepted by peers? The Bayesian model applied here might prove useful for this question, as it enables modelling of relevance of the source of social influence in addition to taste uncertainty (19). Future studies should investigate the ecological selection of influencers and modulation of taste shift, as a function of the quality of earlier development. Bayesian modelling can then dissect the cognitive process of healthy social adaptation, a key requirement of adolescent development, as opposed to the maladaptive influence of undesirable role models.

In our structural brain imaging analysis, we uncovered a neural correlate of taste uncertainty. Using the same social delay discounting task, during functional imaging,we have shown that mPFC expresses neuronal plasticity that predicts preference malleability (27). Myelin maturation unfolds throughout adolescence and into young adulthood and is a key mechanism underlying neuronal plasticity $(42,43)$. This motivated an hypothesis that a marker of myelin in the MPFC would relate to developmental effects on taste uncertainty. Indeed, we found that baseline MT in this region was predictive of a greater reduction in taste uncertainty over time. In contrast, baseline taste uncertainty did not predict changes in MT, and there was no association between the rates of change in both measures. This suggests that the observed longitudinal reduction in taste uncertainty over time is accelerated when myelin in the 
mPFC is at a more mature absolute level, underscoring the importance of brain structural maturation in cognitive development during adolescence. This is consistent with myelination specifically being a key neuro-developmental process relating to important dispositional differences in this period of life (28).

466 Notably, the effect size of the developmental effects on susceptibility to social 467 influence was lower than previously reported in theoretical accounts and lower sample 468 size studies. This is consistent with reports on large-scale replication efforts of original findings in psychological science in bigger sample sizes, which reported only effect sizes on average of $1 / 2$ the originally reported effects (30). On the one hand, it highlights the need for larger sample sizes and replication in order to estimate meaningful effect sizes in the field of developmental psychology. It may, however, also be dependent on precise methodological details and the specific demographics of our sample, e.g., on the fact that our sample did not include very young adolescents for whom strong susceptibility effects have been reported previously $(6,8)$. Our findings stress the importance of longitudinal designs for developmental psychology. Indeed, the association of peer susceptibility with real-life social functioning, as well as neurodevelopmental markers, were only observed within-person, but not across-participant, potentially due to the higher power of within-subject designs as compared to betweensubject designs.

In sum, our study showcases the role of computational modelling and large-scale, longitudinal developmentally sensitive studies $(44,45)$, identifying the psychological mechanisms and neuro-developmental processes which underpin the phenomenon of susceptibility to social influences over adolescent to young adult development. 


\section{Materials and Methods}

\section{Main Sample}

488

489

490

491

492

493

494

495

496

497

498

499

500

501

502

503

504

505

506

507

508

509

The experimental task was delivered as part of a task battery administered to a sample of community dwellers between the ages of 14 and 24 in Cambridgeshire and London, as part of the Neuroscience in Psychiatry Network (NSPN) project (40). All participants provided written informed consent. The Cambridge Central Research Ethics Committee approved the study (12/EE/0250). Data for this task was available from $\mathrm{n}=784$ (401 female) participants for baseline. $\mathrm{N}=738$ of this baseline data has informed a previously published computational model validation paper (25). Participants were 14.10-24.99 years old (mean=19.05, sd=2.96) at baseline. 569 (284 female) participants returned for a second assessment approximately 1.5 years later. Mean age at follow-up was 20.28 years (range: 15.11- 26.48 years, $\mathrm{sd}=2.97$ ) while mean time between first and second assessment was 1.48 years (range: 0.98-2.62 years, $s d=0.30$ ). Structural imaging and task data were available (and passed quality assessment) for $n=184$ participants for both measurement time points (97 females).

\section{Retest Subsample: Testing for training effects in a 6-month follow up subsample of participants}

A subsample of $n=55$ participants completed the task three times, with an additional interim session after a $\sim 6$ month follow-up period. This "retest sample" allows us to index short-term changes (over 6 months), indicative of training effects, from long-term changes (over 1.5 years) indicative of developmental change. 


\section{Computational Modelling}

511 Our model was first introduced and validated on the majority of baseline datasets of 512 this study in (25). In short, we adopt a Bayesian approach to model a change in belief 513 in one's own delay discounting preferences as a function of I) "taste uncertainty" (as 514 reflected in a participants' choice variability), that is how uncertain a person is about 515 their own preferences in the delay discounting task prior to any social exposure in the 516 task and ii) relevance of the social influence source. The model describes that subjects 517 hold a Gaussian belief distribution over their log-discounting coefficient (they are 518 uncertain about their discounting preferences). In previous work (25) we found based 519 on model selection that decision variability during the task was best described as 520 reflecting uncertainty about discounting preference, as opposed to decision noise 521 added after evaluation (as, for example, in the softmax rule). See SI and (25) for 522 details.

\section{Statistical Analysis}

525 All data were analysed using R (46). Mixed models for longitudinal analyses included 526 a categorical within-subject factor 'measurement time point' (baseline vs. follow-up) 527 and a random intercept per subject. They were fit and p-values were calculated based 528 on a Kenward-Roger approximation for degrees-of-freedom using the R package afex 529 (47). In all mixed models, discounting preference of the other was included as a 530 covariate. When analysing social susceptibility, i.e. social shift towards the other, own 531 discounting preference was included as a covariate. All continuous predictors were centred on zero. Post-hoc contrasts were computed using the R package emmeans

533 (48). Latent change score models were fit using the package lavaan (49) with R code 534 provided in (31), freely available at https://osf.io/4bpmq/files/. In all models, we used 535 a robust estimation procedure ('mlr' implemented in lavaan) to account for non536 normality in the data. Plots were generated using ggplot2 (50). Scripts for all statistical 537 analyses are available via https://osf.io/9qu4w/. 


\section{Supplemental Material}

\section{Supplementary Methods}

\section{Task}

We used a social version of a delay discounting task (Figure 1) described in detail elsewhere $(25,27)$. The task consisted of three phases. In phase 1 , participants played 60 trials of a temporal discounting task where they had to decide whether to choose between a smaller amount of money paid out immediately or a larger amount paid out at an indicated time in the future. Phase 1 decisions were used to determine their initial value $k_{\text {phase }}$ in a standard hyperbolic discounting model (51):

$$
V_{D}=\frac{R_{D}}{1+K D}
$$

where $V_{D}$ is the delay-discounted value of a reward, $\mathrm{R}$ is the reward, $\mathrm{D}$ is the delay, and $\mathrm{K}$ is the hyperbolic discounting parameter.

The 60 trials of phase 1 included 30 offer pairs from a standard set covering a wide range of values of $K$. Half of the trials were an interleaved set of 30 from an adaptive algorithm which calculated a probability distribution over possible values of $K$ and then selected a pair of options likely to reduce the entropy of that distribution as much as possible (see (25) for details). Participants were instructed to respond according to their own true preferences.

In phase 2, a second player was introduced. Participants were instructed to make choices in the same delay discounting task for the other player so as to learn the discounting preferences of the other. This preference of the other person was based on the baseline preference of our participant. In a between-subjects manner, the observee's delay discounting preferences was manipulated such that the other was chosen to be either more or less patient than the participant himself. More specifically, in 2/3 of the cases, the observee was chosen to have $k_{\text {other }}$ shifted from $k_{\text {self_phase } 1 \text { by }}$ one standard deviation towards the mean of the population distribution, and in $1 / 3$ of cases away from it (in log space). Participants received feedback as to whether their choice on behalf of the other was correct in terms of the other's discounting preference. where correct choices were defined using a simulation of the other's choice based on their discounting preference. In case the participant's response matched the 
simulation's prediction, the choice was coded as correct. These "learning about the other" trials were presented until either the participant got 8 correct answers out of the most recent 10, or until 60 learning trials were completed.

In phase 3, we interleaved mini-blocks of 10 trials 'choose for self', which were as in phase 1, and 10 trials 'choose for other', in order to keep the other's discounting preference. This allowed us to estimate social shift scores (log $k_{\text {self_phase3 }}-\log$ $k_{\text {self_phase1) }}$ that evaluate a change in delay discounting preference pre- vs. post learning about the other, and thus inter-individual differences in susceptibility to social influence.

We informed participants that one of the 'choose for self' trials from the entire task would be chosen at random and the choice they made paid out for real at the appropriate delay. Participants were instructed that there was no financial incentive to make correct choices in the 'choose for other' trials. The task was programmed in MATLAB 2012a using the Cogent graphics toolbox (http://www.vislab.ucl.ac.uk/Cogent/).

\section{Computational Modeling}

The model assumes that both the subject and the social partner come from the same reference distribution $N\left(k, \sigma^{2}\right)$, the width of which describes the relevance of the other and is a fitted parameter of the model (see supplementary results). By observing the preference choice data of the other, $d_{o}$, subjects can update their own preference belief distribution $p(k)$ in light of what they learn about the other. In this Bayesian formulation, the more uncertain subjects are about their preferences, the more they shift after learning about others. Thus, this model formalizes the notion of informational conformity, namely conforming with others to reduce one's own uncertainty. Please refer to (25) for the algorithmic implementation. In this previous model validation study, we found that participants' behaviour showed evidence for taste 'shifting' correlated to their baseline decision variability, as would be expected if the latter represented uncertainty, upon which Bayesian updating then operated (25). 


\section{Psychometric Measures}

Perceived quality of peer relations

We used the Cambridge Friendship Questionnaire (CFQ) to assess the perceived quality of peer relations (33), a measure available as part of a Home Questionnaire Pack delivered close in time to the in-lab measurements (40). The CFQ assesses the number, and quality of friendships via self-report (e.g. "Do you feel that your friends understand you", "Can you confide in your friends"). Higher scores signify higher satisfaction with peer relations.

\section{MRI pre-processing and Region of Interest extraction}

Of the participants who completed the task, 318 participated in an additional MRI arm of the study. Participants were scanned at T1 $(\mathrm{N}=318)$ and T2 $(\mathrm{N}=235)$ on identical Siemens Magnetom TIM Trio whole-body 3T MRI scanners in Cambridge and London as per the quantitative multi-parameter mapping (MPM) protocol (Weiskopf et al. 2013). This included a whole-brain multi-echo FLASH magnetization transfer weighted contrast at $1 \mathrm{~mm}$ isotropic resolution (TR: 23.7, $\alpha=6^{\circ}, 176$ sagittal slices, FOV=256 $\mathrm{mm} \times 240 \mathrm{~mm}$, matrix $=256 \times 240 \times 176)$. Quantitative magnetization transfer saturation (MT) maps were derived using biophysical models with the hMRI toolbox (www.hmri.info) for SPM (Wellcome Centre for Human Neuroimaging, London, UK, http://www.fil.ion.ucl.ac.uk/spm). These maps have been shown to correlate highly with histological measures of myelin $(52,53)$.

MT maps were spatially pre-processed using a standard pipeline as implemented in the hMRI toolbox. Maps were segmented using unified segmentation (54) and normalised to $\mathrm{MNI}$ space using Diffeomorphic Anatomical Registration using Exponentiated Lie Algebra (DARTEL(55)), followed by spatial smoothing (6mm fullwidth half-maximum) using tissue-weighted smoothing to preserve grey matter / white matter boundaries. Rigorous quality assessment was applied (for details compare(28)) which led to the exclusion of a total of $n=55$ datasets.

We created an anatomically defined mask of the mPFC based on the probabilistic Harvard-Oxford cortical structural atlas (thresholded at 30\%). Mean MT values from within this mask region were extracted from each map using FSL 
(www.fmrib.ox.ac.uk/fsl/) as a proxy for intra-cortical mPFC myelination. Using the same approach, we also extracted mean MT values of the visual cortex (V1) as a control region.

\section{Supplementary Results}

Computational Modelling - 'relevance of the social partner'

Note that apart from taste uncertainty, which is the focus of our developmental study here, also a second parameter, namely 'relevance of the other' ( see (25) and Methods for details) accounts for social shift in our computational model (all $r<-.25$, all $t<-6.24$, all $p<8.4 \mathrm{e}-10)$. There was no significant age correlation with the 'relevance of the other' parameter $(r=.006, t=0.19, d f=780 p=.848)$. Whilst our developmental hypothesis and the current design was indeed focussed on the taste uncertainty parameter of the model, it is interesting to speculate that a different experimental design explicitly manipulating the relevance of the social influence (e.g., as a function of age group (compare, e.g. $(6,8)$ ), might indeed lead to age-related differences in the 'relevance of the social partner' parameter. 
Bivariate latent change score model: Co-development of Social susceptibility and Perceived Quality of Peer Relationships (Cambridge Friendship Questionnaire). est: unstandardized estimate, se: standard error, Cl: confidence interval. est (stand): 542 standardised estimate.

\begin{tabular}{|c|c|c|c|c|c|c|c|}
\hline Parameter & est & se & $\mathbf{z}$ & $\mathbf{p}$ & Cl low & Cl up & $\begin{array}{c}\text { est } \\
\text { (stand) }\end{array}$ \\
\hline \multicolumn{8}{|l|}{ Adults ( $\geq 18)$} \\
\hline $\begin{array}{l}\text { Change Intercept in Social } \\
\text { Susceptibility }\end{array}$ & 0.0401 & 0.5564 & 0.0721 & 0.9425 & -1.0505 & 1.1307 & 0.0215 \\
\hline Mean social Susceptbility at T1 & 0.3940 & 0.0925 & 4.2609 & $<.001$ & 0.2128 & 0.5752 & 0.2706 \\
\hline $\begin{array}{l}\text { Change Variance in Social } \\
\text { Susceptibility }\end{array}$ & 2.1064 & 0.3715 & 5.6704 & $<.001$ & 1.3783 & 2.8345 & 0.6032 \\
\hline Social Susceptbility at T1Variance & 2.1206 & 0.3170 & 6.6891 & $<.001$ & 1.4993 & 2.7420 & 1.0000 \\
\hline Intercept Change in Friendship Quality & 11.6726 & 1.2558 & 9.2953 & $<.001$ & 9.2114 & 14.1338 & 3.4302 \\
\hline Mean Friendship Quality at T1 & 23.2946 & 0.2414 & 96.4908 & $<.001$ & 22.8214 & 23.7678 & 6.1825 \\
\hline Change Variance Friendship Quality & 8.3720 & 0.9609 & 8.7127 & $<.001$ & 6.4887 & 10.2554 & 0.7230 \\
\hline Friendship Quality at T1 Variance & 14.1965 & 1.7565 & 8.0823 & $<.001$ & 10.7538 & 17.6391 & 1.0000 \\
\hline \multicolumn{8}{|l|}{ Regression Paths } \\
\hline $\begin{array}{l}\text { Change in Friendship Quality Social } \\
\text { Susceptibility at T1 }\end{array}$ & 0.0070 & 0.1256 & 0.0561 & 0.9553 & -0.2392 & 0.2532 & 0.0030 \\
\hline $\begin{array}{c}\text { Change in Social Susceptibility } \\
\text { Friendship Quality at T1 }\end{array}$ & 0.0096 & 0.0231 & 0.4141 & 0.6788 & -0.0357 & 0.0548 & 0.0193 \\
\hline $\begin{array}{c}\text { Self Feedback: Change in Friendship } \\
\text { Quality }\end{array}$ & -0.4754 & 0.0518 & -9.1750 & $<.001$ & -0.5770 & -0.3739 & -0.5264 \\
\hline $\begin{array}{l}\text { Self Feedback: Change in Social } \\
\text { Susceptibility }\end{array}$ & -0.8090 & 0.0771 & $\begin{array}{c}- \\
10.4906\end{array}$ & $<.001$ & -0.9602 & -0.6579 & -0.6305 \\
\hline \multicolumn{8}{|l|}{ Covariance Paths } \\
\hline $\begin{array}{c}\text { Social Susceptibility at T1 Friendship } \\
\text { Quality at T1 }\end{array}$ & 0.2496 & 0.3262 & 0.7652 & 0.4441 & -0.3897 & 0.8889 & 0.0455 \\
\hline $\begin{array}{l}\text { Change in Social Susceptibility and } \\
\text { Change in Friendship Quality }\end{array}$ & 0.6662 & 0.2816 & 2.3657 & 0.0180 & 0.1143 & 1.2182 & 0.1586 \\
\hline Adolescents $(<18)$ & & & & & & & \\
\hline
\end{tabular}




\begin{tabular}{|c|c|c|c|c|c|c|c|}
\hline $\begin{array}{c}\text { Intercept Change in Social } \\
\text { Susceptibility }\end{array}$ & 0.5961 & 0.6313 & 0.9442 & 0.3450 & -0.6412 & 1.8335 & 0.3267 \\
\hline Mean social Susceptibility at T1 & 0.7942 & 0.1358 & 5.8486 & $<.001$ & 0.5281 & 1.0604 & 0.5430 \\
\hline $\begin{array}{c}\text { Change Variance in Social } \\
\text { Susceptibility }\end{array}$ & 1.1585 & 0.3369 & 3.4389 & $<.001$ & 0.4982 & 1.8187 & 0.3479 \\
\hline Social Susceptibility at T1 Variance & 2.1391 & 0.4851 & 4.4093 & $<.001$ & 1.1883 & 3.0900 & 1.0000 \\
\hline Intercept Change in Friendship Quality & 12.5330 & 2.0764 & 6.0358 & $<.001$ & 8.4633 & 16.6027 & 3.1627 \\
\hline Mean Friendship Quality at T1 & 22.6078 & 0.3622 & 62.4240 & $<.001$ & 21.8980 & 23.3177 & 5.8212 \\
\hline Change Variance in Friendship Quality & 10.6887 & 2.6383 & 4.0514 & $<.001$ & 5.5177 & 15.8596 & 0.6807 \\
\hline Friendship Quality at T1 Variance & 15.0832 & 2.2863 & 6.5973 & $<.001$ & 10.6022 & 19.5642 & 1.0000 \\
\hline Regression Paths & & & & & & & \\
\hline $\begin{array}{c}\text { Change in Friendship Quality } \\
\text { Social Susceptibility at T1 }\end{array}$ & 0.6240 & 0.2697 & 2.3138 & 0.0207 & 0.0954 & 1.1526 & 0.2303 \\
\hline $\begin{array}{c}\text { Change in Social Susceptibility } \\
\text { Friendship Quality at T1 }\end{array}$ & -0.0025 & 0.0283 & -0.0885 & 0.9295 & -0.0580 & 0.0529 & -0.0053 \\
\hline Self Feedback: Friendship Quality & -0.5132 & 0.0906 & -5.6673 & $<.001$ & -0.6907 & -0.3357 & -0.5030 \\
\hline $\begin{array}{c}\text { Self Feedback: Social Susceptibility } \\
\text { Covariance Paths }\end{array}$ & -1.0079 & 0.0881 & 11.4428 & $<.001$ & -1.1805 & -0.8353 & -0.8078 \\
\hline & & & & & & \\
\hline $\begin{array}{c}\text { Social Susceptibility at T1 Friendship } \\
\text { Quality at T1 }\end{array}$ & -0.3267 & 0.4426 & -0.7380 & 0.4605 & -1.1942 & 0.5409 & -0.0575 \\
\hline $\begin{array}{c}\text { Change in Social Susceptibility and } \\
\text { Change in Friendship Quality }\end{array}$ & -0.2359 & 0.4264 & -0.5533 & 0.5801 & -1.0715 & 0.5997 & -0.0670 \\
\hline
\end{tabular}




\section{S-Table 2}

Latent change score model: Longitudinal development of Social susceptibility and Taste uncertainty. Taste uncertainty covaries with social susceptibility at T1 and longitudinal change in taste uncertainty covaries with longitudinal change in social susceptibility. est: unstandardised estimate, se: standard error, $\mathrm{Cl}$ : confidence interval, est (stand): standardised estimate.

\begin{tabular}{|l|l|c|c|c|c|c|c|}
\hline \multicolumn{1}{|c|}{ Parameter } & est & se & $\mathbf{Z}$ & $\mathbf{p}$ & $\begin{array}{c}\text { Iower } \\
\text { Cl }\end{array}$ & $\begin{array}{c}\text { upper } \\
\text { Cl }\end{array}$ & $\begin{array}{c}\text { est } \\
\text { (stand) }\end{array}$ \\
\hline $\begin{array}{l}\text { Intercept Change in Social } \\
\text { Susceptibility }\end{array}$ & -0.17589 & 0.08154 & -2.1573 & 0.03099 & -0.3357 & -0.01609 & -0.09404 \\
\hline Mean Social Susceptibility at T1 & 0.598969 & 0.06001 & 9.98161 & $<.001$ & 0.481357 & 0.716582 & 0.598969 \\
\hline $\begin{array}{l}\text { Variance Change in Social } \\
\text { susceptibility }\end{array}$ & 3.498715 & 0.39361 & 8.88881 & $<.001$ & 2.727256 & 4.270175 & 1 \\
\hline $\begin{array}{l}\text { Variance Social susceptibility at } \\
\text { T1 }\end{array}$ & 1.925826 & 0.20384 & 9.44770 & $<.001$ & 1.526306 & 2.325347 & 1 \\
\hline $\begin{array}{l}\text { Intercept change in taste } \\
\text { uncertainty }\end{array}$ & -0.1064 & 0.04591 & -2.3173 & 0.0205 & -0.19639 & -0.01641 & -0.09957 \\
\hline Mean taste uncertainty at T1 & 1.276897 & 0.03625 & 35.2251 & $<.001$ & 1.205849 & 1.347945 & 1.276897 \\
\hline $\begin{array}{l}\text { Variance Change in Taste } \\
\text { uncertainty }\end{array}$ & 1.141947 & 0.10468 & 10.9093 & $<.001$ & 0.936786 & 1.347109 & 1 \\
\hline Variance Taste uncertainty at T1 & 0.674559 & 0.06177 & 10.9208 & $<.001$ & 0.553495 & 0.795623 & 0.674559 \\
\hline $\begin{array}{l}\text { Covariation taste uncertainty at } \\
\text { T1 and change in Taste } \\
\text { uncertainty }\end{array}$ & -0.55496 & 0.07682 & -7.2240 & $<.001$ & -0.70553 & -0.40439 & -0.51932 \\
\hline $\begin{array}{l}\text { Covariation Social Susceptibility } \\
\text { at T1 and Change in Social } \\
\text { susceptibility }\end{array}$ & -1.60497 & 0.24836 & -6.4622 & $<.001$ & -2.09175 & -1.11819 & -0.85805 \\
\hline $\begin{array}{l}\text { Covariation Social Susceptibility } \\
\text { at T1 and Taste uncertainty at T1 }\end{array}$ & 0.309783 & 0.04521 & 6.85223 & $<.001$ & 0.221175 & 0.398391 & 0.309783 \\
\hline $\begin{array}{l}\text { Covariation Change in Social } \\
\text { Susceptibility Change in Taste } \\
\text { uncertainty }\end{array}$ & 0.409025 & 0.10722 & 3.81476 & $<.001$ & 0.198874 & 0.619177 & 0.204632 \\
\hline
\end{tabular}




\section{S-Table 3}

Bivariate latent change score model: Co-development of Taste uncertainty and intracortical myelin in mPFC. est: unstandardised estimate, se: standard error, $\mathrm{Cl}$ : confidence interval, est (stand): standardised estimate.

\begin{tabular}{|c|c|c|c|c|c|c|c|}
\hline Parameter & est & se & $\mathbf{z}$ & p & $\begin{array}{c}\text { lower } \\
\text { Cl }\end{array}$ & $\begin{array}{c}\text { upper } \\
\text { Cl }\end{array}$ & $\begin{array}{c}\text { est } \\
\text { (stand) }\end{array}$ \\
\hline Change intercept taste uncertainty & 5.10494 & 1.592517 & 3.205579 & 0.001 & 1.983663 & 8.226217 & 4.572613 \\
\hline Mean taste uncertainty at T1 & $\begin{array}{c}3.09042 \\
1\end{array}$ & 0.606216 & 5.097891 & $<.001$ & 1.90226 & 4.278582 & 3.555317 \\
\hline Change variance taste uncertainty & $\begin{array}{l}0.59380 \\
8\end{array}$ & 0.118516 & 5.010384 & $<.001$ & 0.361522 & 0.826094 & 0.476424 \\
\hline Taste uncertainty at T1 variance & $\begin{array}{c}0.72562 \\
4 \\
\end{array}$ & 0.128672 & 5.639346 & $<.001$ & 0.473432 & 0.977816 & 0.960358 \\
\hline Change intercept mpfc myelin & $\begin{array}{c}0.52287 \\
1\end{array}$ & 0.053562 & 9.761948 & $<.001$ & 0.417891 & 0.627851 & 14.52002 \\
\hline Mean mpfc myelin at $\mathrm{T} 1$ & $\begin{array}{c}0.73812 \\
5\end{array}$ & 0.029111 & 25.35556 & $<.001$ & 0.681068 & 0.795181 & 20.59784 \\
\hline Mpfc myelin change variance & 0.00077 & 0.000105 & 7.308699 & $<.001$ & 0.000563 & 0.000976 & 0.593755 \\
\hline Mpfc myelin variance & $\begin{array}{c}0.00116 \\
2 \\
\end{array}$ & 0.000156 & 7.438966 & $<.001$ & 0.000856 & 0.001468 & 0.904871 \\
\hline \multicolumn{8}{|l|}{ Regression Paths } \\
\hline $\begin{array}{c}\text { Change mpfc myelin mean taste } \\
\text { uncertainty at } \mathrm{T} 1\end{array}$ & 0.00398 & 0.002608 & 1.525937 & $<.001$ & -0.00113 & 0.009092 & 0.096068 \\
\hline $\begin{array}{l}\text { Change taste uncertainty mean } \\
\text { mpfc myelin at T1 }\end{array}$ & $\begin{array}{c}- \\
3.88844\end{array}$ & 1.815342 & -2.14199 & 0.031 & -7.44645 & -0.33044 & -0.12481 \\
\hline Self Feedback mpfc myelin & $\begin{array}{c}- \\
0.63165\end{array}$ & 0.071022 & -8.8938 & $<.001$ & -0.77085 & -0.49245 & -0.62858 \\
\hline Self Feedback taste uncertainty & $\begin{array}{c}- \\
0.91709 \\
\end{array}$ & 0.070924 & -12.9306 & $<.001$ & -1.0561 & -0.77808 & -0.71405 \\
\hline $\begin{array}{l}\text { Covariance Taste uncertainty at T1 } \\
\text { and mpfc myelin at T1 }\end{array}$ & $\begin{array}{c}0.00086 \\
8\end{array}$ & 0.001911 & 0.454303 & 0.645 & -0.00288 & 0.004614 & 0.000868 \\
\hline $\begin{array}{l}\text { Covariance change in taste } \\
\text { uncertainty and change in mpfc } \\
\text { myelin at } \mathrm{T} 1\end{array}$ & 0.00149 & 0.001421 & -1.05187 & 0.293 & -0.00428 & 0.00129 & -0.06988 \\
\hline \multicolumn{8}{|l|}{ Covariates } \\
\hline \multicolumn{8}{|l|}{ Age } \\
\hline Taste uncertainty age & $\begin{array}{c}- \\
3.28956\end{array}$ & 1.925836 & -1.70812 & 0.087 & -7.06413 & 0.485012 & -0.11019 \\
\hline Change in taste uncertainty $\sim$ age & $\begin{array}{c}1.98188 \\
8\end{array}$ & 2.146281 & 0.923406 & 0.356 & -2.22475 & 6.188522 & 0.05169 \\
\hline Myelin at T1 age & $\begin{array}{c}0.25454 \\
1\end{array}$ & 0.094091 & 2.705261 & 0.007 & 0.070126 & 0.438957 & 0.206826 \\
\hline Change in myelin age & $\begin{array}{c}0.07330 \\
1\end{array}$ & 0.076986 & 0.952137 & 0.341 & -0.07759 & 0.224191 & 0.059271 \\
\hline Age variance & $\begin{array}{c}0.00084 \\
8\end{array}$ & $6.50 \mathrm{E}-05$ & 13.03554 & $<.001$ & 0.00072 & 0.000975 & 1 \\
\hline Age intercept & $\begin{array}{c}0.18921 \\
9\end{array}$ & 0.002135 & 88.62727 & $<.001$ & 0.185035 & 0.193404 & 6.498467 \\
\hline \multicolumn{8}{|l|}{ Sex (dummy-coded) } \\
\hline Taste uncertainty $\sim$ sex & $\begin{array}{c}0.04286 \\
2\end{array}$ & 0.123555 & 0.346903 & 0.729 & -0.1993 & 0.285025 & 0.0 \\
\hline Change in taste uncertainty $\sim$ se & $\begin{array}{c}0.19574 \\
5\end{array}$ & 0.113827 & 1.719663 & 0.085 & -0.02735 & 0.418843 & 0.087646 \\
\hline
\end{tabular}




\begin{tabular}{|c|c|c|c|c|c|c|c|}
\hline Myelin at $\mathrm{T} 1 \sim \operatorname{sex}$ & $\begin{array}{c}0.00814 \\
9\end{array}$ & 0.004967 & 1.640642 & $\begin{array}{c}0.1008 \\
72\end{array}$ & -0.00159 & 0.017883 & 0.113671 \\
\hline Change in myelin $\sim$ sex & $\begin{array}{c}0.00916 \\
4\end{array}$ & 0.004113 & 2.228238 & $\begin{array}{c}0.0258 \\
65\end{array}$ & 0.001103 & 0.017224 & 0.127208 \\
\hline Sex variance & $\begin{array}{c}0.24988 \\
4\end{array}$ & 0.000788 & 317.0144 & $<.001$ & 0.248339 & 0.251429 & 1 \\
\hline Sex intercept & $\begin{array}{c}0.48924 \\
7\end{array}$ & 0.036653 & 13.34797 & $<.001$ & 0.417408 & 0.561086 & 0.978721 \\
\hline \multicolumn{8}{|l|}{ IQ } \\
\hline Taste uncertainty $\sim \mathrm{IQ}$ & -1.1602 & 0.467127 & -2.48369 & 0.013 & -2.07575 & -0.24465 & -0.15027 \\
\hline Change in taste uncertainty $\sim \mathrm{IQ}$ & $\begin{array}{c}- \\
1.32668 \\
\end{array}$ & 0.468555 & -2.83142 & 0.005 & -2.24503 & -0.40833 & -0.13379 \\
\hline Myelin at $\mathrm{T} 1 \sim \mathrm{IQ}$ & $\begin{array}{c}0.00643 \\
6\end{array}$ & 0.021587 & 0.298161 & 0.766 & -0.03587 & 0.048746 & 0.020221 \\
\hline Change in myelin $\sim \mathrm{IQ}$ & $\begin{array}{c}- \\
0.03075 \\
\end{array}$ & 0.015901 & -1.93363 & 0.061 & -0.06191 & 0.000419 & -0.09613 \\
\hline $\mathrm{IQ}$ variance & $\begin{array}{c}0.01267 \\
5\end{array}$ & 0.0013 & 9.749059 & $<.001$ & 0.010127 & 0.015224 & 1 \\
\hline IQ intercept & $\begin{array}{c}1.10865 \\
6\end{array}$ & 0.008258 & 134.2551 & $<.001$ & 1.092471 & 1.124841 & 9.847329 \\
\hline \multicolumn{8}{|l|}{ Site (dummy-coded) } \\
\hline Myelin at $\mathrm{T} 1 \sim$ site 1 at $\mathrm{T} 1$ & $\begin{array}{c}- \\
0.01503\end{array}$ & 0.006667 & -2.25512 & 0.024 & -0.0281 & -0.00197 & -0.14548 \\
\hline Myelin at T1 site 2 at T1 & $\begin{array}{c}- \\
0.01614 \\
\end{array}$ & 0.007867 & -2.05094 & 0.040 & -0.03155 & -0.00072 & -0.15614 \\
\hline Myelin at T2 site at T2 & $\begin{array}{c}- \\
0.00178\end{array}$ & 0.004757 & -0.37521 & 0.708 & -0.01111 & 0.007538 & -0.01906 \\
\hline Site 1 at $\mathrm{T} 1$ variance & $\begin{array}{c}0.12024 \\
5\end{array}$ & 0.018318 & 6.56445 & $<.001$ & 0.084343 & 0.156147 & 1 \\
\hline Site 2 at $\mathrm{T} 1$ variance & $\begin{array}{c}0.12024 \\
5 \\
\end{array}$ & 0.018318 & 6.56445 & $<.001$ & 0.084343 & 0.156147 & 1 \\
\hline Site at T2 variance & $\begin{array}{c}0.11634 \\
3 \\
\end{array}$ & 0.018287 & 6.3621 & $<.001$ & 0.080501 & 0.152185 & 1 \\
\hline Site 1 at T1 intercept & $\begin{array}{c}0.13978 \\
5\end{array}$ & 0.025426 & 5.497727 & $<.001$ & 0.089951 & 0.189619 & 0.403113 \\
\hline Site2 at T1 intercept & $\begin{array}{c}0.13978 \\
5\end{array}$ & 0.025426 & 5.497727 & $<.001$ & 0.089951 & 0.189619 & 0.403113 \\
\hline Site at T2 intercept & $\begin{array}{c}0.13440 \\
9\end{array}$ & 0.02501 & 5.374196 & $<.001$ & 0.08539 & 0.183427 & 0.394055 \\
\hline \multicolumn{8}{|l|}{ Residual Covariances } \\
\hline Age IQ & $\begin{array}{c}0.00040 \\
1\end{array}$ & 0.000231 & 1.733565 & 0.082 & $\begin{array}{c}-5.24 \mathrm{E}- \\
05\end{array}$ & 0.000854 & 0.122298 \\
\hline Sex IQ & $\begin{array}{c}- \\
0.00198\end{array}$ & 0.004081 & -0.48616 & 0.623 & -0.00998 & 0.006014 & -0.03525 \\
\hline Site1 at T1 Site at T1 & $\begin{array}{c}- \\
0.01954 \\
\end{array}$ & 0.0046 & -4.24791 & $<.001$ & -0.02856 & -0.01052 & -0.1625 \\
\hline Site1 at T1 Site at T2 & 0.11562 & 0.018188 & 6.357108 & $<.001$ & 0.079973 & 0.151267 & 0.977531 \\
\hline Site2 at T1 Site at T2 & $\begin{array}{c}- \\
0.01879 \\
\end{array}$ & 0.004484 & -4.19015 & $<.001$ & -0.02758 & -0.01 & -0.15885 \\
\hline
\end{tabular}




\section{S-Table 4}

Neuroscience in Psychiatry Network (NSPN) Consortium author list

\begin{tabular}{|l|}
\hline NSPN Principle Investigators \\
\hline Edward Bullmore \\
Raymond Dolan \\
Ian Goodyer \\
Peter Fonagy \\
\hline NSPN (funded) staff: \\
\hline Michael Moutoussis \\
Tobias Hauser \\
Sharon Neufeld \\
Rafael Romero-Garcia \\
Michelle St Clair \\
Petra Vértes \\
Kirstie Whitaker \\
Becky Inkster \\
Gita Prabhu \\
Cinly Ooi \\
Umar Toseeb \\
Barry Widmer \\
Junaid Bhatti \\
Laura Villis \\
Ayesha Alrumaithi \\
Sarah Birt \\
Aislinn Bowler \\
Kalia Cleridou \\
Hina Dadabhoy \\
Emma Davies \\
Ashlyn Firkins \\
Sian Granville \\
Elizabeth Harding \\
\hline
\end{tabular}




\begin{tabular}{|l|}
\hline Janchai King \\
Danae Kokorikou \\
Christina Maurice \\
Cleo McIntosh \\
Jessica Memarzia \\
Harriet Mills \\
Ciara O'Donnell \\
Sara Pantaleone \\
Jenny Scott \\
Andrea Reiter \\
Lucy Vanes \\
\hline
\end{tabular}




\section{S-Figure 1}
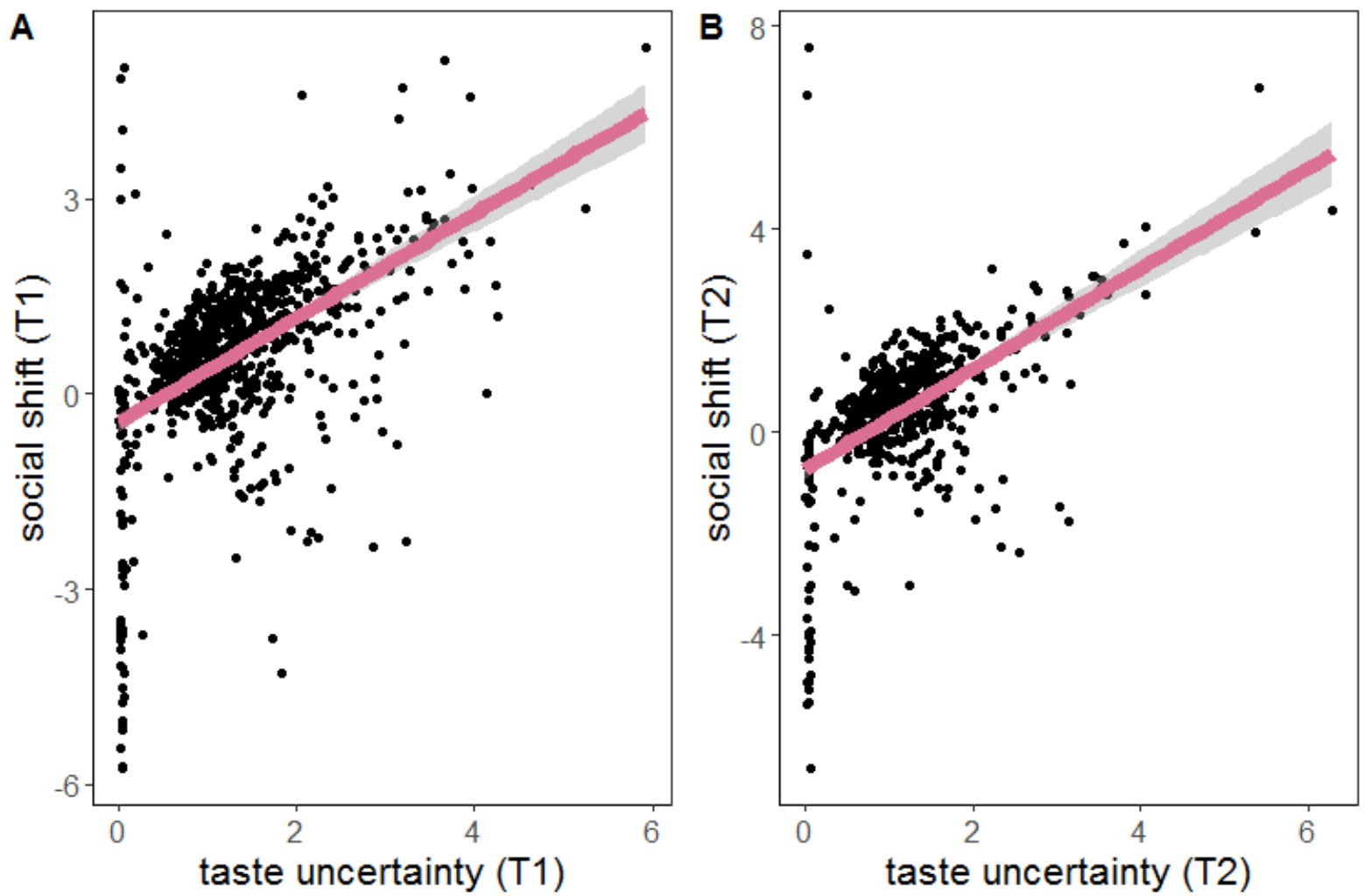

Taste uncertainty significantly predicted social susceptibility at both T1 and T2, in line with an informational account of conformity(22). 
544 1. Romer D, et al. (1994) Social Influences on the Sexual-Behavior of Youth at Risk for Hiv $545 \quad$ Exposure. American Journal of Public Health 84(6):977-985.

546 2. Weitzman ER, Nelson TF, \& Wechsler H (2003) Taking up binge drinking in college: the

3. Blakemore S-J \& Mills KL (2014) Is adolescence a sensitive period for sociocultural processing? Annual review of psychology 65:187-207.

4. Foulkes L \& Blakemore S-J (2016) Is there heightened sensitivity to social reward in adolescence? Current opinion in neurobiology 40:81-85.

5. Knoll L, Leung JT, Foulkes L, \& Blakemore S-J (2017) Age-related differences in social influence on risk perception depend on the direction of influence. Journal of Adolescence 60:53-63.

6. Knoll L, Magis-Weinberg L, Speekenbrink M, \& Blakemore S-J (2015) Social influence on risk perception during adolescence. Psychological science:0956797615569578.

7. Costanzo PR \& Shaw ME (1966) Conformity as a function of age level. Child development:967975.

8. Reiter AMF, Suzuki S, O'Doherty JP, Li SC, \& Eppinger B (2019) Risk contagion by peers affects learning and decision-making in adolescents. J Exp Psychol Gen Advance online publication.

9. Len-Rios ME, Hughes HE, McKee LG, \& Young HN (2016) Early adolescents as publics: A national survey of teens with social media accounts, their media use preferences, parental mediation, and perceived Internet literacy. Public Relat Rev 42(1):101-108.

10. Mckay HD (1962) Social Influence on Adolescent Behavior. Jama-Journal of the American Medical Association 182(6):643-\&.

11. Reyna VF \& Farley F (2006) Risk and Rationality in Adolescent Decision Making: Implications for Theory, Practice, and Public Policy. Psychol Sci Public Interest 7(1):1-44.

12. Albert D \& Steinberg L (2011) Peer influences on adolescent risk behavior. Inhibitory control and drug abuse prevention, (Springer), pp 211-226.

13. Loke AY \& Mak Y-w (2013) Family process and peer influences on substance use by adolescents. International journal of environmental research and public health 10(9):38683885.

14. Johnston KL \& White KM (2003) Binge-drinking: A test of the role of group norms in the theory of planned behaviour. Psychol Health 18(1):63-77.

15. Lundborg P (2006) Having the wrong friends? Peer effects in adolescent substance use. Journal of health economics 25(2):214-233.

16. Tsakpinoglou F \& Poulin F (2017) Best friends' interactions and substance use: The role of friend pressure and unsupervised co-deviancy. Journal of adolescence 60:74-82.

17. Sawyer SM, et al. (2012) Adolescence: a foundation for future health. Lancet 379(9826):16301640 .

18. Niederkrotenthaler T, et al. (2019) Association of Increased Youth Suicides in the United States With the Release of 13 Reasons Why. JAMA Psychiatry Published online May 29, 2019. doi:10.1001/jamapsychiatry.2019.0922.

19. Large I, Pellicano E, Mojzisch A, \& Krug K (2019) Developmental trajectory of social influence integration into perceptual decisions in children. Proceedings of the National Academy of Sciences of the United States of America 116(7):2713-2722.

20. Casey BJ, et al. (2011) Behavioral and neural correlates of delay of gratification 40 years later. Proc Natl Acad Sci U S A 108(36):14998-15003.

21. Goschke T (2014) Dysfunctions of decision-making and cognitive control as transdiagnostic mechanisms of mental disorders: advances, gaps, and needs in current research. International journal of methods in psychiatric research 23(S1):41-57.

22. Toelch U \& Dolan RJ (2015) Informational and Normative Influences in Conformity from a Neurocomputational Perspective. Trends Cogn Sci 19(10):579-589. 
23. Deutsch M \& Gerard HB (1955) A study of normative and informational social influences upon individual judgment. The journal of abnormal social psychology 51(3):629.

24. Somerville LH (2013) The teenage brain sensitivity to social evaluation. Current directions in psychological science 22(2):121-127.

25. Moutoussis M, Dolan RJ, \& Dayan P (2016) How People Use Social Information to Find out What to Want in the Paradigmatic Case of Inter-temporal Preferences. Plos Computational Biology 12(7):e1004965.

26. Revelt D \& Train K (2000) Customer-specific taste parameters and mixed logit: Households' choice of electricity supplier. (UCB).

27. Garvert MM, Moutoussis M, Kurth-Nelson Z, Behrens TE, \& Dolan RJ (2015) Learning-induced plasticity in medial prefrontal cortex predicts preference malleability. Neuron 85(2):418-428.

28. Ziegler G, et al. (2019) Compulsivity and impulsivity traits linked to attenuated developmental fronto-striatal myelination trajectories. Nature Neuroscience 22(6):992.

29. Nicolle A, et al. (2012) An agent independent axis for executed and modeled choice in medial prefrontal cortex. Neuron 75(6):1114-1121.

30. Collaboration OS (2015) Estimating the reproducibility of psychological science. Science 349(6251):aac4716.

31. Kievit RA, et al. (2018) Developmental cognitive neuroscience using latent change score models: A tutorial and applications. Developmental Cognitive Neuroscience 33:99-117.

32. van Harmelen AL, et al. (2016) Friendships and Family Support Reduce Subsequent Depressive Symptoms in At-Risk Adolescents. PLoS One 11(5):e0153715.

33. van Harmelen AL, et al. (2017) Adolescent friendships predict later resilient functioning across psychosocial domains in a healthy community cohort. Psychol Med 47(13):2312-2322.

34. Whelan R, et al. (2014) Neuropsychosocial profiles of current and future adolescent alcohol misusers. Nature 512(7513):185-189.

35. Prinstein MJ, Boergers J, \& Spirito A (2001) Adolescents' and their friends' health-risk behavior: Factors that alter or add to peer influence. J Pediatr Psychol 26(5):287-298.

36. White SF, et al. (2014) Reward: empirical contribution. Temporal discounting and conduct disorder in adolescents. J Pers Disord 28(1):5-18.

37. St Clair MC, et al. (2017) Characterising the latent structure and organisation of self-reported thoughts, feelings and behaviours in adolescents and young adults. PLoS One 12(4):e0175381.

38. lacobucci D, Saldanha N, \& Deng XY (2007) A meditation on mediation: Evidence that structural equations models perform better than regressions. Journal of Consumer Psychology 17(2):139-153.

39. Hu LT \& Bentler PM (1999) Cutoff Criteria for Fit Indexes in Covariance Structure Analysis: Conventional Criteria Versus New Alternatives. Structural Equation Modeling-a Multidisciplinary Journal 6(1):1-55.

40. Kiddle B, et al. (2017) Cohort profile: the NSPN 2400 Cohort: a developmental sample supporting the Wellcome Trust NeuroScience in Psychiatry Network. International journal of epidemiology 47(1):18-19g.

41. Fonagy $P$ \& Luyten $P$ (2018) Conduct problems in youth and the RDoC approach: $A$ developmental, evolutionary-based view. Clin Psychol Rev 64:57-76.

42. Fields RD (2005) Myelination: an overlooked mechanism of synaptic plasticity? Neuroscientist 11(6):528-531.

43. Fields RD (2015) A new mechanism of nervous system plasticity: activity-dependent myelination. Nat Rev Neurosci 16(12):756-767.

44. Bolenz F, Reiter AM, \& Eppinger B (2017) Developmental changes in learning: Computational mechanisms and social influences. Frontiers in Psychology 8:2048.

45. Hauser TU, Will GJ, Dubois M, \& Dolan RJ (2019) Annual Research Review: Developmental computational psychiatry. J Child Psychol Psychiatry 60(4):412-426. 
46. RDevelopment CORE TEAM R (2008) R: A language and environment for statistical computing. ( $R$ foundation for statistical computing Vienna, Austria).

47. Singmann H, Bolker B, Westfall J, \& Aust FJRpv (2015) afex: Analysis of factorial experiments.

48. Lenth R (2018) Emmeans: Estimated marginal means, aka least-squares means. 1(1).

49. Rosseel Y, et al. (2018) Package 'lavaan'.

50. Wickham H (2016) ggplot2: elegant graphics for data analysis (Springer).

51. McClure SM, Ericson KM, Laibson DI, Loewenstein G, \& Cohen JD (2007) Time discounting for primary rewards. J Neurosci 27(21):5796-5804.

52. Schmierer K, Scaravilli F, Altmann DR, Barker GJ, \& Miller DH (2004) Magnetization transfer ratio and myelin in postmortem multiple sclerosis brain. Ann Neurol 56(3):407-415.

53. Turati $L$, et al. (2015) In vivo quantitative magnetization transfer imaging correlates with histology during de- and remyelination in cuprizone-treated mice. NMR Biomed 28(3):327337.

54. Ashburner J \& Friston KJ (2005) Unified segmentation. Neuroimage 26(3):839-851.

55. Ashburner J (2007) A fast diffeomorphic image registration algorithm. Neuroimage 38(1):95113. 